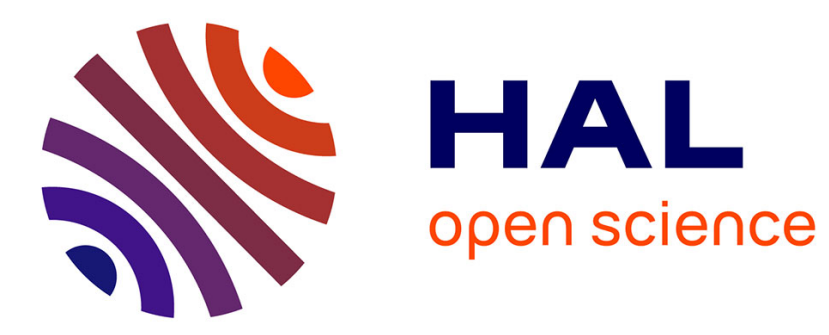

\title{
The role of the Pauli principle in spin exchange collisions
}

M. Pinard, F. Laloë

\section{To cite this version:}

M. Pinard, F. Laloë. The role of the Pauli principle in spin exchange collisions. Journal de Physique, 1980, 41 (8), pp.769-797. 10.1051/jphys:01980004108076900 . jpa-00209302

\section{HAL Id: jpa-00209302 https://hal.science/jpa-00209302}

Submitted on 1 Jan 1980

HAL is a multi-disciplinary open access archive for the deposit and dissemination of scientific research documents, whether they are published or not. The documents may come from teaching and research institutions in France or abroad, or from public or private research centers.
L'archive ouverte pluridisciplinaire HAL, est destinée au dépôt et à la diffusion de documents scientifiques de niveau recherche, publiés ou non, émanant des établissements d'enseignement et de recherche français ou étrangers, des laboratoires publics ou privés. 


\title{
The role of the Pauli principle in spin exchange collisions
}

\author{
M. Pinard and F. Laloë \\ Laboratoire de Spectroscopie Hertzienne de l'E.N.S. (*), 24, rue Lhomond, F 75231 Paris, France
}

(Reçu le 20 novembre 1979, accepté le 14 avril 1980)

\begin{abstract}
Résumé. - Nous discutons le rôle des collisions d'échange de spin sur les variables atomiques internes (orientation, alignement, etc.) en insistant sur le rôle précis du principe de Pauli. Le calcul s'applique à des particules discernables ou à des particules identiques, sans prendre comme point de départ un hamiltonien effectif d'interaction d'échange. On évalue l'opérateur densité d'un atome après collision sous une forme opératorielle qui facilite la discussion de divers phénomènes physiques. Le premier terme qui apparaît dans l'évolution de l'opérateur densité est un terme de transfert qui existe, que les particules soient discernables ou identiques. Les termes suivants sont des conséquences spécifiques du principe d'antisymétrisation de Pauli, appliqué aux électrons et aux noyaux. Ces termes peuvent être décrits au moyen de champs magnétiques (ou gradients électriques) fictifs électroniques ou nucléaires. De plus, on obtient des termes dont l'origine est l'échange combiné des électrons et des noyaux, en particulier l'échange global d'atomes (qui sont des bosons dans le cas de l'hydrogène). Les équations d'évolution des opérateurs densité sont appliquées à quelques cas particuliers : découplage hyperfin nul ou total, résonances Zeeman ou hyperfines,...
\end{abstract}

\begin{abstract}
The effect of spin exchange collisions on the internal variables (orientation, alignment, etc.) of colliding atoms is discussed, with particular emphasis on the role played by the Pauli principle. The calculation presented is valid for distinguishable or identical particles, and the initial introduction of an effective exchange hamiltonian for the spins is avoided. The one-atom density matrix of the internal variables is calculated after collision, in an operatorial form which enables the discussion of various physical effects. The first term in the density matrix evolution is a transfer term which does not originate from particle indistinguishability. The following terms are indeed introduced by the Pauli antisymmetrization principle applied to electrons and nuclei, and they can be described as electronic or nuclear apparent magnetic field (or electric gradient). In addition, terms arising from the combined exchange of electrons and nuclei are obtained, in particular from the exchange of identical whole atoms (bosons in the case of hydrogen). The evolution equations for the density operators are applied to a few particular cases in order to evaluate spin exchange effects in various situations : weak or strong hyperfine decoupling, Zeeman or hyperfine resonances, etc...
\end{abstract}

1. Introduction. - The importance of exchange collisions in atomic physics is well known. Spin exchange collisions have been used in many optical pumping experiments in order to transfer spin polarization from one atomic species to a different one, when direct optical orientation of the latter was not feasible [1,2]. Spin exchange collisions also strongly affect the hydrogen maser operation, since they broaden and shift the hyperfine transition of the atoms [3, 4]. This phenomenon also occurs with the rubidium maser $[5,6]$. Spin exchange processes affect the intensity of absorption or emission of the $21 \mathrm{~cm}$

$\left(^{*}\right)$ Laboratoire ‘issocié au Centre National de la Recherche Scientifique. hydrogen line in radioastronomy, and this phenomenon stimulated some of the first calculations on the subject [7].

It is clear that the Pauli principle (or symmetrization principle if bosons are involved) plays an important role in the evaluation of the effect of spin exchange collisions. Most quantum mechanics textbooks discuss the effect of particle identity on collision phenomena, but the emphasis is generally put on how symmetry interference effects modify the spatial distribution of the scattered particles. In optical pumping experiments, what is really important is the evolution of the internal atomic variables (orientation, alignment, etc...). Usually, an average is taken over all atomic velocities, before and after the collision. The aim of this article is precisely to focus interest on the evo- 
lution of the density matrix describing the internal atomic variables, and to discuss carefully the effects of particle identity (electrons or nuclei).

There are already several articles in the literature dealing with spin exchange collisions (see for example references [7-13] or the references given in [1, 2]). The point of view generally adopted is that, since the two electrons are identical particles, the spin singlet state $(S=0)$ must correspond to a bonding potential curve for the transient $\mathrm{H}-\mathrm{H}$ molecule, and their spin triplet state $(S=1)$ to an antibonding potential curve. This correlation between the spin states of the electrons and their orbital binding energy can be accounted for by using a phenomenological exchange hamiltonian acting on the spins. Since we precisely wish to study the effect of particle identity on spin exchange processes, we adopt a different approach. First, we ignore the electron indistinguishability and perform a complete calculation of the evolution of the internal variables. In the second stage, we take into account identity effects for electrons and nuclei, and discuss the new terms which appear in the equations. Another difference between the calculation presented here and previous literature arises from the way the equations are written. For example, in an important article, L. C. Balling, R. J. Hanson and F. M. Pipkin [9] discuss spin exchange collisions in hydrogen and explicitely calculate the internal variable density matrix for a system of two atoms. Since hydrogen atoms possess 4 Zeeman and hyperfine sublevels in the ground state, $16 \times 16$ density matrices are needed (see also [11]). In the case of deuterium atoms, $36 \times 36$ matrices would appear. Here we shall rather calculate the evolution of the one-atom density matrix $(4 \times 4$ matrix for hydrogen) in the general case, and keep an operatorial formulation which is not limited to a particular state basis (coupled or decoupled basis for example : the same equations are valid in a zero magnetic field or in a high field producing complete hyperfine decoupling). Our approach simplifies the discussion of the physical meaning of the various terms obtained. For example, the electron identity term emerges directly as an apparent magnetic field term proportional to the electronic spin polarization. We shall particularly insist on the effects of nuclear indistinguishability, which are not discussed in detail in the literature (and sometimes simply ignored). These effects introduce additional terms in the equation of evolution of the density operators. One of them is similar to the electron indistinguishability term and introduces a nuclear apparent magnetic field, another term accounts for the fact that atoms as a whole are identical particles (bosons in the case of hydrogen atoms), etc...

The organization of this paper is the following. In section 2, the one-atom density matrix calculation is given, first for distinguishable particles, then for identical electrons and finally for identical electrons and identical protons. At each step, a physical dis- cussion of the various terms obtained is given. In section 3 , the equations are applied to a few cases of interest (calculation of the spin exchange shift in various circumstances). Phase shift calculations, which can be completely avoided as long as no numerical calculation of the cross sections is needed, are recalled in an appendix. This article is intended to be self contained and accessible to a reader not already familiar with the literature on the subject.

2. Calculation of the one-atom density operator. In this section, we study the effect of a collision between two hydrogen atoms on their internal variables. These variables are related to the existence of a non-zero electronic and nuclear spin for each atom. We first ignore the fact that both electrons and both nuclei (protons for $\mathbf{H}$, deutons for $\mathrm{D}$ ) are identical particles, and perform a calculation of the density matrix where each electron and each proton is labelled by a number, 1 or 2 . Later we introduce the identity effects of the electrons in order to see how the equations are modified. Finally, the same process is repeated to account for the nucleus identity effects.

No particular hypothesis is necessary concerning the value of the spin of each particle. The nuclear spin of the colliding atoms may be 1/2 (hydrogen), 1 (deuterium), etc... The calculation is actually valid for any alkali atom, although in this case each atom possesses several core electrons in addition to the external valence electron. Since the electronic core forms a complete shell (zero angular momentum), it actually plays no essential role in this problem. For the same reason, the calculation remains unchanged for collisions between identical ions having one valence electron (or hole). In principle, the electron spin could also have any value in the following discussion (although this value must of course be the same for both atoms), but this generalization is rather academic; even for exotic hydrogenic systems, the particle playing the role of the electron is generally a spin $1 / 2$ (or spin 0) particle.

\subsection{Distinguishable PARTICleS. - 2.1.1 Nota-} tion. - Let us consider two atoms, the first one formed by electron $e_{1}$ and nucleus $n_{1}$, the second one by electron $e_{2}$ and nucleus $n_{2}$. Both atoms are in the ground state, and $\left|\mathrm{e}_{1}: \varphi_{\mathrm{n}_{i}}\right\rangle(i=1,2)$ is the ket in the external variable state space of electron 1 when it is bound to nucleus $\mathrm{n}_{i} ;\left|\mathrm{e}_{2}: \varphi_{\mathrm{n}_{i}}\right\rangle(i=1,2)$ is the same state for electron $\mathrm{e}_{2}$. Both electrons also have spin variables, and $\left|\mathrm{e}_{1}: M_{S}\right\rangle$ is, in the spin state space of $\mathrm{e}_{1}$, the eigenstate of the projection $S_{1 z}$ of its $\operatorname{spin} \mathbf{S}_{1}$ on an arbitrary quantization axis $\mathrm{O} z\left(M_{S}= \pm 1 / 2\right.$ for an electron). The spin states of $e_{2}$ are noted in a similar way. In addition to these electronic degrees of freedom, the system under study also possesses nuclear variables. The nuclear spin variables kets can be written $\left|n_{1,2}: m_{I}\right\rangle$ where $m_{I} \hbar$ is the eigenvalue of $I_{1 z}$ or $I_{2 z}$, projection of the nuclear spin $I_{1}$ of $n_{1}$ or $I_{2}$ of $n_{2}$ on the $\mathrm{O} z$ axis. 
Finally, external nuclear variables have to be included. Throughout the present article, we shall study collision effects in the centre of mass reference frame of the two atoms. In this frame, the external state of the two atoms is written $\left|n_{1}: \mathbf{k} ; n_{2}:-\mathbf{k}\right\rangle$ where $\hbar k$ is the linear momentum of the $n_{1}$ atom, - $\hbar \mathbf{k}$ the linear momentum of the $\mathrm{n}_{2}$ atom.

No correlation is supposed to exist initially between the external and internal variables of each of the two atoms approaching the collision. In the same way, no initial correlation is assumed between the internal (spin) variables of the two atoms (this is an excellent approximation in low density gases). Under these conditions, the density operator of the whole system before collision can be written :

$$
\begin{gathered}
\sigma_{\text {init. }}=\int \mathrm{d}^{3} k_{\mathrm{i}} u\left(\mathbf{k}_{\mathrm{i}}\right) \int \mathrm{d}^{3} k_{\mathrm{i}}^{\prime} u^{*}\left(\mathbf{k}_{\mathrm{i}}^{\prime}\right) \\
\left|\mathrm{n}_{1}: \mathbf{k}_{\mathrm{i}} ; \mathrm{n}_{2}:-\mathbf{k}_{\mathrm{i}}\right\rangle\left\langle\mathrm{n}_{1}: \mathbf{k}_{\mathrm{i}}^{\prime} ; \mathrm{n}_{2}:-\mathbf{k}_{\mathrm{i}}^{\prime}|\otimes| \mathrm{e}_{1}: \varphi_{\mathrm{n}_{1}}\right\rangle\left\langle\mathrm{e}_{1}: \varphi_{\mathrm{n}_{1}}|\otimes| \mathrm{e}_{2}: \varphi_{\mathrm{n}_{2}}\right\rangle\left\langle\mathrm{e}_{2}: \varphi_{\mathrm{n}_{2}}\right| \otimes \\
\otimes \rho_{1}\left(\mathrm{n}_{1}, \mathrm{e}_{1}\right) \otimes \rho_{2}\left(\mathrm{n}_{2}, \mathrm{e}_{2}\right) .
\end{gathered}
$$

In this expression, $u\left(\mathbf{k}_{\mathbf{i}}\right)$ is a complex wave function associated with a wave packet in the space of the two atom relative particle (spanned by the kets $\left|\mathrm{n}_{1}: \mathbf{k} ; \mathrm{n}_{2}:-\mathbf{k}\right\rangle$ ). We assume that $u\left(\mathbf{k}_{\mathrm{i}}\right)$ is normalized :

$$
\int \mathrm{d}^{3} k_{\mathrm{i}} u^{*}\left(\mathbf{k}_{\mathrm{i}}\right) u\left(\mathbf{k}_{\mathrm{i}}\right)=1 .
$$

Since we want to include the possibility of any type of correlation between the internal variables of $e_{1}$ and $\mathrm{n}_{1}$ on one hand, $\mathrm{e}_{2}$ and $\mathrm{n}_{2}$ on the other, we have introduced the two density operators $\rho_{1}\left(\mathrm{e}_{1}, \mathrm{n}_{1}\right)$ and $\rho_{2}\left(\mathrm{e}_{2}, \mathrm{n}_{2}\right)\left({ }^{1}\right)$. These operators describe the initial internal variable state of the two atoms and, in general, are not a product of an electronic by a nuclear spin variable density matrix (they act in the state space spanned by the kets $\left|\mathrm{e}_{1}: M_{S}\right\rangle \otimes\left|\mathrm{n}_{1}: m_{I}\right\rangle$ and $\left|\mathrm{e}_{2}: M_{S}\right\rangle \otimes\left|\mathrm{n}_{2}: m_{I}\right\rangle$ respectively). In the particular case of two hydrogen atoms ( $1 / 2$ electronic and nuclear spins), $\rho_{1}$ and $\rho_{2}$ correspond to $4 \times 4$ matrices.

We assume that $\rho_{1}$ and $\rho_{2}$ are normalized so that :

$$
\operatorname{Tr}\left\{\rho_{1}\right\}=\operatorname{Tr}\left\{\rho_{2}\right\}=1 .
$$

2.1.2 Evolution operator. - We denote by $S$ the unitary operator which describes the evolution between time $-t$ (long before the atoms interact) and $+t$ (long after the collision). Since the collision time $\tau_{\mathrm{c}}$ is very short (typically $\tau_{\mathrm{c}} \simeq 10^{-12} \mathrm{~s}$ ) and the main interaction between atoms is electrostatic, it is possible to neglect completely the spin evolution during the collision. This implies that the various magnetic fields (external fields, hyperfine coupling, relativistic effects during the collision) acting on the spins are not too strong $\left({ }^{2}\right)$. Such a condition is generally well fulfilled in most laboratory experiments $\left({ }^{3}\right)$. Neglecting the effects of collisions on spins is an approximation which is very commonly used in atomic collision studies. When electronic spins are involved, it is often referred to as the Wigner rule. In the case of nuclear spins, the approximation is generally even better, and has been introduced by P. L. Bender in his thesis [14] (the nuclear spin inertia in a collision has sometimes been called a spin Franck-Condon effect [15] or also nuclear flywheel effect).

The matrix elements of $S$ between initial and final states are of two different types. The first type includes elements like :

$$
\left[\left\langle\mathrm{n}_{1}: \mathbf{k}_{\mathrm{f}} ; \mathrm{n}_{2}:-\mathbf{k}_{\mathrm{f}}\left|\left\langle\mathrm{e}_{1}: \varphi_{\mathrm{n}_{1}}\left|\left\langle\mathrm{e}_{2}: \varphi_{\mathrm{n}_{2}}\right|\right] S\left[\left|\mathrm{n}_{1}: \mathbf{k}_{\mathrm{i}} ; \mathrm{n}_{2}:-\mathbf{k}_{\mathrm{i}}\right\rangle\left|\mathrm{e}_{1}: \varphi_{\mathrm{n}_{1}}\right\rangle\left|\mathrm{e}_{2}: \varphi_{\mathrm{n}_{2}}\right\rangle\right]=S_{\mathrm{d}}\left(\mathbf{k}_{\mathrm{f}}, \mathbf{k}_{\mathrm{i}}\right)\right.\right.\right.\right.
$$

( ${ }^{1}$ ) The letter $\sigma$ will be used for density operators acting in the state space of the 2 atoms (external and internal variables). The letter $\rho$ will refer to one-atom internal variable density operators (reduced operators).

$\left({ }^{2}\right)$ This can be true even if the hyperfine structure in the ground state of the hydrogen atoms is completely decoupled.

$\left({ }^{3}\right)$ Even when very small, the evolution of the spins during a collision is not always completely negligible. In the case of spin exchange collision, very high precision experiments with the hydrogen maser have made it possible to observe the effect of the collision duration $\tau_{\mathrm{c}}[16]$. In this article, we shall completely neglect these very small effects and consider that $S$ is an operator which acts only in the external variable state space of the particles. 
or, if spin variables are involved :

$$
\begin{gathered}
{\left[\left\langle\mathrm{n}_{1}: \mathbf{k}_{\mathrm{f}} ; \mathrm{n}_{2}:-\mathbf{k}_{\mathrm{f}}\right|\left\langle\mathrm{n}_{1}: m_{I}\right|\left\langle\mathrm{n}_{2}: m_{I}^{\prime}\right|\left\langle\mathrm{e}_{1}: \varphi_{\mathrm{n}_{1}}, M_{S}\right|\left\langle\mathrm{e}_{2}: \varphi_{\mathrm{n}_{2}}, M_{S}^{\prime}\right|\right]} \\
S\left[\left|\mathrm{e}_{1}: \varphi_{\mathrm{n}_{1}}, M_{S}^{\prime \prime}\right\rangle\left|\mathrm{e}_{2}: \varphi_{\mathrm{n}_{2}}, M_{S}^{\prime \prime \prime}\right\rangle\left|\mathrm{n}_{1}: m_{I}^{\prime \prime}\right\rangle\left|\mathrm{n}_{2}: m_{I}^{\prime \prime \prime}\right\rangle\left|\mathrm{n}_{1}: \mathbf{k}_{\mathrm{i}} ; \mathrm{n}_{2}:-\mathbf{k}_{\mathrm{i}}\right\rangle\right]= \\
\quad=S_{\mathrm{d}}\left(\mathbf{k}_{\mathrm{f}}, \mathbf{k}_{\mathrm{i}}\right) \delta_{m_{I} m_{I}^{\prime \prime}} \delta_{m_{I}^{\prime} m_{I}^{\prime \prime}} \delta_{M_{S} M_{S}^{\prime \prime}} \delta_{M_{S}^{\prime} M_{S}^{\prime \prime \prime}}
\end{gathered}
$$

( $S$ does not affect spin variables). In the initial as well as the final state, $e_{1}$ is bound to $n_{1}$ and $e_{2}$ to $n_{2}$.

Since $e_{1}$ and $e_{2}$ behave in the same way during the collision, one can write :

$$
\begin{gathered}
S_{\mathrm{d}}\left(\mathbf{k}_{\mathrm{f}}, \mathbf{k}_{\mathrm{i}}\right)=\left[\left\langle\mathrm{n}_{1}: \mathbf{k}_{\mathrm{f}} ; \mathrm{n}_{2}:-\mathbf{k}_{\mathrm{f}}\right|\left\langle\mathrm{e}_{1}: \varphi_{\mathrm{n}_{2}}\right|\left\langle\mathrm{e}_{2}: \varphi_{\mathrm{n}_{1}}\right|\right] \\
S\left[\left|\mathrm{n}_{1}: \mathbf{k}_{\mathrm{i}} ; \mathrm{n}_{2}:-\mathbf{k}_{\mathrm{i}}\right\rangle\left|\mathrm{e}_{1}: \varphi_{\mathrm{n}_{2}}\right\rangle\left|\mathrm{e}_{2}: \varphi_{\mathrm{n}_{1}}\right\rangle\right] .
\end{gathered}
$$

Also, the fact that $n_{1}$ and $n_{2}$ behave in the same way allow us to write :

$$
S_{\mathrm{d}}\left(\mathbf{k}_{\mathrm{f}}, \mathbf{k}_{\mathrm{i}}\right)=S_{\mathrm{d}}\left(-\mathbf{k}_{\mathrm{f}},-\mathbf{k}_{\mathrm{i}}\right) .
$$

We shall call all corresponding processes the direct processes. They are characterized by the fact that the association between the numbered protons and electrons are not changed by the collision.

It is clear that other processes are also possible. We assume that all physical properties of $e_{1}$ and $e_{2}$ which affect the collision are exactly the same (although $e_{1}$ and $e_{2}$ are here considered as distinguishable particles), and the same holds for $\mathrm{n}_{1}$ and $\mathrm{n}_{2}$. Therefore, the two final states :

$$
\begin{aligned}
& \left|\mathrm{n}_{1}: \mathbf{k}_{\mathrm{f}} ; \mathrm{n}_{2}:-\mathbf{k}_{\mathrm{f}}\right\rangle\left|\mathrm{n}_{1}: m_{I}\right\rangle\left|\mathrm{n}_{2}: m_{I}^{\prime}\right\rangle\left|\mathrm{e}_{1}: \varphi_{\mathrm{n}_{1}}, M_{S}\right\rangle\left|\mathrm{e}_{2}: \varphi_{\mathrm{n}_{2}}, M_{S}^{\prime}\right\rangle \\
& \left|\mathrm{n}_{1}: \mathbf{k}_{\mathrm{f}} ; \mathrm{n}_{2}:-\mathbf{k}_{\mathrm{f}}\right\rangle\left|\mathrm{n}_{1}: m_{I}\right\rangle\left|\mathrm{n}_{2}: m_{I}^{\prime}\right\rangle\left|\mathrm{e}_{1}: \varphi_{\mathrm{n}_{2}}, M_{S}\right\rangle\left|\mathrm{e}_{2}: \varphi_{\mathrm{n}_{1}}, M_{S}^{\prime}\right\rangle
\end{aligned}
$$

( $e_{1}$ is bound to $n_{2}$ and $e_{2}$ to $n_{1}$ in the second state) have exactly the same energy (exchange degeneracy). Both states can therefore be reached after collision, and we introduce the notation :

$$
\begin{gathered}
S_{\mathrm{t}}\left(\mathbf{k}_{\mathrm{f}}, \mathbf{k}_{\mathrm{i}}\right)=\left[\left\langle\mathrm{n}_{1}: \mathbf{k}_{\mathrm{f}} ; \mathrm{n}_{2}:-\mathbf{k}_{\mathrm{f}}\right|\left\langle\mathrm{e}_{1}: \varphi_{\mathrm{n}_{2}}\right|\left\langle\mathrm{e}_{2}: \varphi_{\mathrm{n}_{1}}\right|\right] S \\
{\left[\left|\mathrm{n}_{1}: \mathbf{k}_{\mathrm{i}} ; \mathrm{n}_{2}:-\mathbf{k}_{\mathrm{i}}\right\rangle\left|\mathrm{e}_{1}: \varphi_{\mathrm{n}_{1}}\right\rangle\left|\mathrm{e}_{2}: \varphi_{\mathrm{n}_{2}}\right\rangle\right]}
\end{gathered}
$$

(an analogous relationship including spin variables can easily be written as in (3b). One can also write :

and

$$
\begin{gathered}
S_{\mathrm{t}}\left(\mathbf{k}_{\mathrm{f}}, \mathbf{k}_{\mathrm{i}}\right)=\left[\left\langle\mathrm{n}_{1}: \mathbf{k}_{\mathrm{f}} ; \mathrm{n}_{2}:-\mathbf{k}_{\mathrm{f}}\right|\left\langle\mathrm{e}_{1}: \varphi_{\mathrm{n}_{1}}\right|\left\langle\mathrm{e}_{2}: \varphi_{\mathrm{n}_{2}}\right|\right] S \\
{\left[\left|\mathrm{n}_{1}: \mathbf{k}_{\mathrm{i}} ; \mathrm{n}_{2}:-\mathbf{k}_{\mathrm{i}}\right\rangle\left|\mathrm{e}_{1}: \varphi_{\mathrm{n}_{2}}\right\rangle\left|\mathrm{e}_{2}: \varphi_{\mathrm{n}_{1}}\right\rangle\right]}
\end{gathered}
$$

$$
S_{\mathrm{t}}\left(\mathbf{k}_{\mathrm{f}}, \mathbf{k}_{\mathrm{i}}\right)=S_{t}\left(-\mathbf{k}_{\mathrm{f}},-\mathbf{k}_{\mathrm{i}}\right) \text {. }
$$

The corresponding processes are called transfer processes $\left({ }^{4}\right)$.

Physically, the possibility that electron $e_{1}$ becomes bound to $n_{2}$ and that $e_{2}$ transfers to $n_{1}$ can for example be understood by considering $n_{1}$ and $n_{2}$ as two classical particles fixed at two points $\mathbf{R}_{1}$ and $\mathbf{R}_{2}$ respectively (in the Born-Oppenheimer approximation, the motion of $n_{1}$ and $n_{2}$ can be studied in a second step). Electrons $e_{1}$ and $e_{2}$ are then attracted by two potential wells centred at $\mathbf{R}_{1}$ and $\mathbf{R}_{2}$. At very large internuclear distances $\left(\left|\mathbf{R}_{1}-\mathbf{R}_{2}\right| \gg a_{0}\right.$, Bohr radius), two degenerate states of the 2 electron system occur :

$$
\begin{aligned}
& \left|\mathrm{e}_{1}: \varphi_{\mathbf{R}_{1}}\right\rangle\left|\mathrm{e}_{2}: \varphi_{\mathbf{R}_{2}}\right\rangle \\
& \left|\mathrm{e}_{2}: \varphi_{\mathbf{R}_{1}}\right\rangle\left|\mathrm{e}_{1}: \varphi_{\mathbf{R}_{2}}\right\rangle
\end{aligned}
$$

( $e_{1}$ is bound in the $\mathbf{R}_{1}$ potential well, $e_{2}$ in the $\mathbf{R}_{2}$ potential well, or the reverse). Actually $(5 a, b)$ are not strictly speaking stationary states, since the tunnel effect allows both electrons to transfer from one potential well to the other $\left({ }^{5}\right)$.

At lower values of the internuclear distance, the tunnel effect is no longer negligible, the coupling between the states removes the energy degeneracy, and an energy difference $\Delta E$ between the $g$ and $u$ states appears.

(4) They are sometimes called exchange processes since they correspond to collisions where $\mathrm{n}_{1}$ and $\mathrm{n}_{2}$ exchange their numbered electrons. Nevertheless, we shall reserve the word exchange for effects related to the Pauli principle (particle indistinguishability).

${ }^{5}$ ) Due to the interaction between $e_{1}$ and $e_{2}$, the states in which $e_{1}$ and $e_{2}$ are both bound to the same nucleus have a much higher energy than the initial state; they are not considered here. 
Starting from the initial state $(5 a)$, the system will be found in the state (5b) after a time $T=\pi \hbar / \Delta E$. If now $\left|\mathbf{R}_{1}-\mathbf{R}_{2}\right|$ is small enough to allow $e_{1}$ and $e_{2}$ to go classically from one well to the other, the transfer time can be even shorter.

To emphasize that the transfer probability does not in itself include any electron identity effect, one can consider a single electron problem : a collision between a proton and an hydrogen atom. In this simple case also, the electron can jump from one potential well to the other. The probability of this process can be expressed in terms of $\mathrm{g}$ and $\mathrm{u}$ potentials of the $\mathrm{H}_{2}^{+}$ion.

A more precise evaluation of the transfer probability can be obtained by calculating the energies $E_{\mathrm{g}}(R)$ and $E_{\mathrm{u}}(R)$ of the two electronic stationary states as a function of the internuclear distance $R=\left|\mathbf{R}_{1}-\mathbf{R}_{2}\right|$ (Born-Oppenheimer approximation), and using the phase shift method. The principle of this calculation is recalled in Appendix A.

2.1.3 Internal variable density operator after collision. - Let us first calculate the spin density matrix of $e_{1}$ and $n_{1}$ if, after collision, $e_{1}$ remains bound to $n_{1}$ (in other words, we only consider the projection of the final state through the operator $\left.\left|\mathrm{e}_{1}: \varphi_{\mathrm{n}_{1}} ; \mathrm{e}_{2}: \varphi_{\mathrm{n}_{2}}\right\rangle\left\langle\mathrm{e}_{1}: \varphi_{\mathrm{n}_{1}} ; \mathrm{e}_{2}: \varphi_{n_{2}}\right|\right)$. We must therefore take a partial trace over the external variables and over the spin variables of $\mathrm{e}_{2}$ and $\mathrm{n}_{2}$. The result is the density matrix $\rho_{\mathrm{f}}\left(\mathrm{e}_{1}, \mathrm{n}_{1}\right)$ with elements :

$$
\begin{aligned}
& \left\langle m_{I}, M_{S}\left|\rho_{\mathrm{f}}\left(\mathrm{n}_{1}, \mathrm{e}_{1}\right)\right| m_{I}^{\prime}, M_{S}^{\prime}\right\rangle=\sum_{m_{I}^{\prime \prime}} \sum_{M_{S}^{\prime \prime}} \int \mathrm{d}^{3} k_{\mathrm{f}} \\
& {\left[\left\langle\mathrm{n}_{1}: \mathbf{k}_{\mathrm{f}} ; \mathrm{n}_{2}:-\mathbf{k}_{\mathrm{f}}\left|<\mathrm{n}_{1}: m_{I}\right|<\mathrm{n}_{2}: m_{I}^{\prime \prime}\left|<\mathrm{e}_{1}: \varphi_{\mathrm{n}_{1}}, M_{S}\right|<\mathrm{e}_{2}: \varphi_{\mathrm{n}_{2}}, M_{S}^{\prime \prime}\right|\right]} \\
& S \sigma_{\text {init. }} S^{+} \\
& {\left[\left|\mathrm{n}_{1}: \mathbf{k}_{\mathrm{f}} ; \mathrm{n}_{2}:-\mathbf{k}_{\mathrm{f}}\right\rangle\left|\mathrm{n}_{1}: m_{I}^{\prime}\right\rangle\left|\mathrm{n}_{2}: m_{I}^{\prime \prime}\right\rangle\left|\mathrm{e}_{1}: \varphi_{\mathrm{n}_{1}}, M_{S}^{\prime}\right\rangle\left|\mathrm{e}_{2}: \varphi_{\mathrm{n}_{2}}, M_{S}^{\prime \prime}\right\rangle\right]} \\
& =\sum_{m_{I}^{\prime \prime}} \sum_{M_{S}} \int \mathrm{d}^{3} k_{\mathrm{i}} u\left(\mathbf{k}_{\mathrm{i}}\right) \int \mathrm{d}^{3} \mathbf{k}_{\mathrm{i}}^{\prime} u^{*}\left(\mathbf{k}_{\mathrm{i}}^{\prime}\right) \int \mathrm{d}^{3} k_{\mathrm{f}} S_{\mathrm{d}}\left(\mathbf{k}_{\mathrm{f}}, \mathbf{k}_{\mathrm{i}}\right) S_{\mathrm{d}}^{*}\left(\mathbf{k}_{\mathrm{f}}, \mathbf{k}_{\mathrm{i}}^{\prime}\right) \\
& \left\langle m_{I} M_{S}\left|\rho_{1}\right| m_{I}^{\prime} M_{S}^{\prime}\right\rangle\left\langle m_{I}^{\prime \prime} M_{S}^{\prime \prime}\left|\rho_{2}\right| m_{I}^{\prime \prime} M_{S}^{\prime \prime}\right\rangle \text {. }
\end{aligned}
$$

The trace of $\rho_{2}$ is equal to one. Then, if we set :

$$
A_{\mathrm{d}}=\int \mathrm{d}^{3} k_{\mathrm{i}} u\left(\mathbf{k}_{\mathrm{i}}\right) \int \mathrm{d}^{3} k_{\mathrm{i}}^{\prime} u^{*}\left(\mathbf{k}_{\mathrm{i}}^{\prime}\right) \int \mathrm{d}^{3} k_{\mathrm{f}} S_{\mathrm{d}}\left(\mathbf{k}_{\mathrm{f}}, \mathbf{k}_{\mathrm{i}}\right) S_{\mathrm{d}}^{*}\left(\mathbf{k}_{\mathrm{f}}, \mathbf{k}_{\mathrm{i}}^{\prime}\right),
$$

we merely obtain :

$$
\rho_{\mathrm{f}}\left(\mathrm{n}_{1}, \mathrm{e}_{1}\right)=A_{\mathrm{d}} \rho_{1}
$$

In a similar way, one gets :

$$
\rho_{\mathrm{f}}\left(\mathrm{n}_{2}, \mathrm{e}_{2}\right)=A_{\mathrm{d}} \rho_{2} .
$$

Physically, these equalities are easy to interpret : since the collision does not change the spin states, when $e_{1}$ remains associated with $\mathrm{n}_{1}$ or $\mathrm{e}_{2}$ with $\mathrm{n}_{2}$, their internal state density operator is not modified at all.

The other possibility is that $e_{1}$ ends up in the $\left|\varphi_{n_{2}}\right\rangle$ state, $e_{2}$ in the $\left|\rho_{n_{1}}\right\rangle$ state. Let us for example calculate the internal density operator of $n_{1}$ and $e_{2}$ when these two particles are bound after collision. We obtain :

$$
\begin{aligned}
&\left\langle m_{I} M_{S}\left|\rho_{\mathrm{f}}\left(\mathrm{n}_{1}, \mathrm{e}_{2}\right)\right| m_{I}^{\prime} M_{S}^{\prime}\right\rangle=\sum_{m_{I}^{\prime \prime}} \sum_{M_{S}^{\prime \prime}} \int \mathrm{d}^{3} k_{\mathrm{f}} \\
& {\left[\left\langle\mathrm{n}_{1}: \mathbf{k}_{\mathrm{f}} ; \mathrm{n}_{2}:-\mathbf{k}_{\mathrm{f}}\right|\left\langle\mathrm{n}_{1}: m_{I}\right|\left\langle\mathrm{n}_{2}: m_{I}^{\prime \prime}\right|\left\langle\mathrm{e}_{1}: \varphi_{\mathrm{n}_{2}}, M_{S}^{\prime \prime}\right|\left\langle\mathrm{e}_{2}: \varphi_{\mathrm{n}_{1}}, M_{S}\right|\right] } \\
& S \sigma_{\text {init. }} S^{+} \\
& {\left[\left|\mathrm{n}_{1}: \mathbf{k}_{\mathrm{f}} ; \mathrm{n}_{2}:-\mathbf{k}_{\mathrm{f}}\right\rangle\left|\mathrm{n}_{1}: m_{I}^{\prime}\right\rangle\left|\mathrm{n}_{2}: m_{I}^{\prime \prime}\right\rangle\left|\mathrm{e}_{1}: \varphi_{\mathrm{n}_{2}}, M_{S}^{\prime \prime}\right\rangle\left|\mathrm{e}_{2}: \varphi_{\mathrm{n}_{1}}, M_{S}^{\prime}\right\rangle\right] } \\
&=\sum_{m_{I}^{\prime \prime}} \sum_{M_{S}^{\prime \prime}} \int \mathrm{d}^{3} k_{\mathrm{i}} u\left(\mathbf{k}_{\mathrm{i}}\right) \int \mathrm{d}^{3} k_{\mathrm{i}}^{\prime} u^{*}\left(\mathbf{k}_{\mathrm{i}}^{\prime}\right) \int \mathrm{d}^{3} k_{\mathrm{f}} S_{\mathrm{t}}\left(\mathbf{k}_{\mathrm{f}}, \mathbf{k}_{\mathrm{i}}\right) S_{\mathrm{t}}^{*}\left(\mathbf{k}_{\mathrm{f}}, \mathbf{k}_{\mathrm{i}}^{\prime}\right) \\
&\left\langle m_{I} M_{S}^{\prime \prime}\left|\rho_{1}\right| m_{I}^{\prime} M_{S}^{\prime \prime}\right\rangle\left\langle m_{I}^{\prime \prime} M_{S}\left|\rho_{2}\right| m_{I}^{\prime \prime} M_{S}^{\prime}\right\rangle \\
&=A_{\mathrm{t}} \sum_{M_{S}^{\prime \prime}}\left\langle m_{I} M_{S}^{\prime \prime}\left|\rho_{1}\right| m_{I}^{\prime} M_{S}^{\prime \prime}\right\rangle \sum_{m_{I}^{\prime \prime}}\left\langle m_{I}^{\prime \prime} M_{S}\left|\rho_{2}\right| m_{I}^{\prime \prime} M_{S}^{\prime}\right\rangle
\end{aligned}
$$

where :

$$
A_{\mathrm{t}}=\int \mathrm{d}^{3} k_{\mathrm{i}} u\left(\mathbf{k}_{\mathrm{i}}\right) \int \mathrm{d}^{3} k_{\mathrm{i}}^{\prime} u^{*}\left(\mathbf{k}_{\mathrm{i}}^{\prime}\right) \int \mathrm{d}^{3} k_{\mathrm{f}} S_{\mathrm{t}}\left(\mathbf{k}_{\mathrm{f}}, \mathbf{k}_{\mathrm{i}}\right) S_{\mathrm{t}}^{*}\left(\mathbf{k}_{\mathrm{f}}, \mathbf{k}_{\mathrm{i}}^{\prime}\right)
$$


We therefore obtain :

$$
\rho_{\mathrm{f}}\left(\mathrm{n}_{1}, \mathrm{e}_{2}\right)=A_{\mathrm{t}} \operatorname{Tr}_{\mathrm{S}}\left\{\rho_{1}\right\} \otimes \operatorname{Tr}_{I}\left\{\rho_{2}\right\} .
$$

where $\operatorname{Tr}_{S}$ denotes a partial trace over the $\mathbf{S}$ variables, $\operatorname{Tr}_{I}$ a partial trace over the $\mathbf{I}$ variables. Equation (11) shows that, if $e_{2}$ is bound to $n_{1}$ after collision, the internal variable density matrix of the corresponding atom is a (tensor) product of nuclear and electronic spin density matrices (no correlation between the two spins).

Using the fact that $S$ is an unitary operator $\left(S^{+} S=1\right)$ and relation (2), one can easily show from definitions (7) and (10) that :

$$
A_{\mathrm{t}}+A_{\mathrm{d}}=1 .
$$

In the following, we shall therefore replace $A_{\mathrm{d}}$ by $1-A_{\mathrm{t}}$. Another way to obtain equality (12) is to calculate the spin density operator of $\mathrm{n}_{1}$ after collision, which is, according to (8a) and (11) :

$$
\rho_{\mathrm{f}}\left(\mathrm{n}_{1}\right)=\left(A_{\mathrm{d}}+A_{\mathrm{t}}\right) \operatorname{Tr}_{S}\left\{\rho_{1}\right\} .
$$

Since this operator must have a trace equal to one, (12) is necessarily true.

If now, in the experiment considered, the particles $\mathrm{e}_{1}$ and $\mathrm{e}_{2}$ play equivalent roles (although we still consider here that they are theoretically distinguishable), it is convenient to define the density operator of the atom labelled by nucleus $n_{1}$ as :

$$
\rho(\text { atom } 1)=\rho\left(\mathrm{n}_{1}, \mathrm{e}_{1}\right)+\rho\left(\mathrm{n}_{1}, \mathrm{e}_{2}\right) .
$$

In the same way, the density operator of the atom labelled by $n_{2}$ is :

$$
\rho(\operatorname{atom} 2)=\rho\left(\mathrm{n}_{2}, \mathrm{e}_{1}\right)+\rho\left(\mathrm{n}_{2}, \mathrm{e}_{2}\right) .
$$

Each collision between an atom of species 1 and an atom of species 2 changes $\rho$ (atom 1) into :

$$
\rho_{\mathrm{f}}(\text { atom } 1)=\left(1-A_{1}\right) \rho_{1}+A_{\mathrm{t}} \operatorname{Tr}_{S}\left\{\rho_{1}\right\} \otimes \operatorname{Tr}_{I}\left\{\rho_{2}\right\}
$$

[one can easily check on this result that $\operatorname{Tr}\left\{\rho_{\mathrm{f}}\right.$ atom 1$\left.)\right\}=1$ when equations $(2 b)$ are satisfied]. Let us for example consider an ensemble of atoms of one isotope ( $\mathrm{n}_{1}$ type nuclei, internal density operator $\left.\rho_{1}\right)$, undergoing collisions with atoms of another isotope $\left(\mathrm{n}_{2}\right.$ type nuclei, internal density operator $\left.\rho_{2}\right)\left({ }^{6}\right)$. Without taking into account Pauli's antisymmetrization principle for the electrons exchanged, we then find that the density operator evolution due to spin exchange collisions is :

$$
\left.\frac{\mathrm{d}}{\mathrm{d} t} \rho_{1}\right|_{\text {coll. } 1-2} \propto \rho_{\mathrm{f}}(\text { atom } 1)-\rho_{1}
$$

or

$$
\left.\frac{\mathrm{d}}{\mathrm{d} t} \rho_{1}\right|_{\text {coll. } 1-2}=\frac{1}{T_{\text {coll. }}^{(1)}}\left[-\rho_{1}+\operatorname{Tr}_{S}\left\{\rho_{1}\right\} \otimes \operatorname{Tr}_{I}\left\{\rho_{2}\right\}\right]
$$

In this expression, $1 / T_{\mathrm{coll}}^{(1)}$ is a rate constant which is proportional to the average over relative velocities $v$ of the product $v A_{\mathrm{t}}$ for all possible collisions (average over energies and initial velocity directions), and also proportional to the number density of atoms 2 . Equations (16) show how collisions between atoms of type 1 and atoms of type 2 transfer the electronic orientation from species 2 to species 1 . It is clear that the density operator evolution of species 2 evolves under the effect of the same collisions according to :

$$
\left.\frac{\mathrm{d}}{\mathrm{d} t} \rho_{2}\right|_{\text {coll. } 1-2}=\frac{1}{T_{\text {coll. }}^{(2)}}\left[-\rho_{2}+\operatorname{Tr}_{S}\left\{\rho_{2}\right\} \otimes \operatorname{Tr}_{I}\left\{\rho_{1}\right\}\right]
$$

with :

$$
\frac{T_{\text {coll. }}^{(1)}}{T_{\text {coll. }}^{(2)}}=\frac{\mathrm{n}_{1}}{\mathrm{n}_{2}}
$$

where $\mathrm{n}_{1}\left(\right.$ resp. $\left.\mathrm{n}_{2}\right)$ is the number density of atoms 1 (resp. atoms 2 ).

$\left({ }^{6}\right)$ At this stage, the nuclear spin $I_{1}$ and $I_{2}$ of $\mathrm{n}_{1}$ and $\mathrm{n}_{2}$ can have different values. 
It is also possible to calculate the effect of collisions between atoms of the same species, having initially the same internal density operator. To do this, we assume that $\rho_{1}=\rho_{2}=\rho$, and the same reasoning gives :

$$
\left.\frac{\mathrm{d}}{\mathrm{d} t} \rho\right|_{\text {coll. }}=\frac{1}{T_{\text {coll. }}}\left[-\rho+\operatorname{Tr}_{S}\{\rho\} \otimes \operatorname{Tr}_{I}\{\rho\}\right]
$$

This equation shows how spin exchange collisions tend to suppress the correlations between the electronic and nuclear spin variables. One must nevertheless keep in mind that, here, we have only considered the effect of the collisions on the density operator $\rho$. It is clear that, during the time between collisions, the hyperfine coupling hamiltonian modifies the internal state of the atoms and can re-establish strong correlation between the spins (in other words, $\mathbf{I}$ and $\mathbf{S}$ recouple after collisions).

2.2 INDISTINGUISHABLE ELECTRONS. - In this section, we consider that the two particles $\mathrm{e}_{1}$ and $\mathrm{e}_{2}$ exchanged by nuclei $n_{1}$ and $n_{2}$, are two indistinguishable electrons, and therefore introduce totally antisymmetric wave functions (Pauli principle) to describe them. We denote by $P_{\mathrm{e}}$ the exchange operator between these two electrons. For example :

$$
P_{\mathrm{e}}\left|\mathrm{e}_{1}: \varphi_{\mathrm{n}_{1}}, M_{S} ; \mathrm{e}_{2}: \varphi_{\mathrm{n}_{2}}, M_{S}^{\prime}\right\rangle=\left|\mathrm{e}_{1}: \varphi_{\mathrm{n}_{2}}, M_{S}^{\prime} ; \mathrm{e}_{2}: \varphi_{\mathrm{n}_{1}}, M_{S}\right\rangle
$$

We still assume that $n_{1}$ and $n_{2}$ are fundamentally distinguishable particles, even if the experiment considered do not allow to distinguish between them (the role of nuclear identity of the 2 atoms will be studied in the next section).

2.2.1 Density operator after collision. - The density operator of the system before collision is now :

$$
\frac{1}{\sqrt{2}}\left[1-P_{\mathrm{e}}\right] \sigma_{\text {init. }} \frac{1}{\sqrt{2}}\left[1-P_{\mathrm{e}}\right]
$$

where $\sigma_{\text {init. }}$ is given by equation (1). After collision, it becomes :

$$
\frac{1}{2} S\left[1-P_{\mathrm{e}}\right] \sigma_{\text {init. }}\left[1-P_{\mathrm{e}}\right] S^{+}
$$

We now want to calculate the density operator $\rho_{\mathrm{f}}\left(\mathrm{n}_{1}, \mathrm{e}\right)$ associated, after collision, with the internal variables of the atom labelled by $n_{1}$. To do this, we have to take the trace of the operator written above over all internal variables of the $\mathrm{n}_{2}$-atom (quantum numbers $m_{I}^{\prime \prime}, M_{S}^{\prime \prime}$ ) and over $\mathbf{k}_{\mathrm{f}}$. Therefore, $\rho_{\mathrm{f}}\left(\mathrm{n}_{1}, \mathrm{e}\right)$ is given by :

$$
\begin{aligned}
\left\langle m_{I}, M_{S}\left|\rho_{\mathrm{f}}\left(\mathrm{n}_{1}, \mathrm{e}\right)\right| m_{I}^{\prime}, M_{S}^{\prime}\right\rangle=\int \mathrm{d}^{3} k_{\mathrm{i}} u\left(\mathbf{k}_{\mathrm{i}}\right) \int \mathrm{d}^{3} k_{\mathrm{i}}^{\prime} u^{*}\left(\mathbf{k}_{\mathrm{i}}^{\prime}\right) \int \mathrm{d}^{3} k_{\mathrm{f}} \sum_{m_{I}^{\prime \prime}} \sum_{M_{S}^{\prime \prime}} \\
{\left[\left\langle\mathrm{n}_{1}: \mathbf{k}_{\mathrm{f}} ; \mathrm{n}_{2}:-\mathbf{k}_{\mathrm{f}}\right|\left\langle\mathrm{n}_{1}: m_{I}\right|\left\langle\mathrm{n}_{2}: m_{I}^{\prime \prime}\right|\left\langle\mathrm{e}_{1}: \varphi_{\mathrm{n}_{1}}, M_{S}\right|\left\langle\mathrm{e}_{2}: \varphi_{\mathrm{n}_{2}}, M_{S}^{\prime \prime}\right|\right] } \\
{\left[1-P_{\mathrm{e}}\right] S \sigma_{\text {init. }} S^{+}\left[1-P_{\mathrm{e}}\right] } \\
{\left[\left|\mathrm{n}_{1}: \mathbf{k}_{\mathrm{f}} ; \mathrm{n}_{2}:-\mathbf{k}_{\mathrm{f}}\right\rangle\left|\mathrm{n}_{1}: m_{I}^{\prime}\right\rangle\left|\mathrm{n}_{2}: m_{I}^{\prime \prime}\right\rangle\left|\mathrm{e}_{1}: \varphi_{\mathrm{n}_{1}}, M_{S}^{\prime}\right\rangle\left|\mathrm{e}_{2}: \varphi_{\mathrm{n}_{2}}, M_{S}^{\prime \prime}\right\rangle\right] . }
\end{aligned}
$$

To obtain this expression, one can consider fully antisymmetrized final states (after collision). The operator in the centre of the right hand side of (19) is then actually replaced by :

$$
\left[\frac{1-P_{\mathrm{e}}}{\sqrt{2}}\right] S\left[\frac{1-P_{\mathrm{e}}}{\sqrt{2}}\right] \sigma_{\text {init. }}\left[\frac{1-P_{\mathrm{e}}}{\sqrt{2}}\right] S^{+}\left[\frac{1-P_{\mathrm{e}}}{\sqrt{2}}\right] \text {. }
$$

But, since $S$ and $S^{+}$commute with $P_{\mathrm{e}}$, and $P_{\mathrm{e}}^{2}=1$, one can easily obtain (19). It is also possible to write (19) directly by using the fact that, when the initial state vector of the system has been fully antisymmetrized, it is no longer indispensable to perform another antisymmetrization of the kets associated with the measurements (see exercice 8 of complement D-XIV, Ref. [17]).

Four different terms appear in (19) : one term without any $P_{\mathrm{e}}$ operator, one with two such operators, and two terms containing one $P_{\mathrm{e}}$ either on the right or the left side. It is easily seen that the first of these four terms is exactly the one which was calculated in (6). It is therefore equal to :

$$
\left(1-A_{\mathrm{t}}\right)\left\langle m_{I}, M_{S}\left|\rho_{1}\right| m_{I}^{\prime} M_{S}^{\prime}\right\rangle \text {. }
$$


The term which contains both $P_{\mathrm{e}}$ operators is nothing but the expression calculated in (9). It thus gives a contribution :

$$
A_{\mathrm{t}}\left\langle m_{I}, M_{S}\left|\operatorname{Tr}_{S}\left\{\rho_{1}\right\} \otimes \operatorname{Tr}_{I}\left\{\rho_{2}\right\}\right| m_{I}^{\prime}, M_{S}^{\prime}\right\rangle
$$

The two terms which contain $P_{\mathrm{e}}$ only once are new. To write them, let us introduce the coefficient :

$$
C_{S}=\int \mathrm{d}^{3} k_{\mathrm{i}} u\left(\mathbf{k}_{\mathrm{i}}\right) \int \mathrm{d}^{3} k_{\mathrm{i}}^{\prime} u^{*}\left(\mathbf{k}_{\mathrm{i}}^{\prime}\right) \int \mathrm{d}^{3} k_{\mathrm{f}} S_{\mathrm{d}}\left(\mathbf{k}_{\mathrm{f}}, \mathbf{k}_{\mathrm{i}}\right) S_{\mathrm{t}}^{*}\left(\mathbf{k}_{\mathrm{f}}, \mathbf{k}_{\mathrm{i}}^{\prime}\right)
$$

The contribution to (19) of these two terms is :

and

$$
-C_{S} \sum_{m_{I}^{\prime \prime}} \sum_{M_{S}^{\prime \prime}}\left\langle m_{I}, M_{S}\left|\rho_{1}\right| m_{I}^{\prime}, M_{S}^{\prime \prime}\right\rangle\left\langle m_{I}^{\prime \prime}, M_{S}^{\prime \prime}\left|\rho_{2}\right| m_{I}^{\prime \prime}, M_{S}^{\prime}\right\rangle
$$

$$
-C_{S}^{*} \sum_{m_{I}^{\prime \prime}} \sum_{M_{S}^{\prime \prime}}\left\langle m_{I}, M_{S}^{\prime \prime}\left|\rho_{1}\right| m_{I}^{\prime}, M_{S}^{\prime}\right\rangle\left\langle m_{I}^{\prime \prime}, M_{S}\left|\rho_{2}\right| m_{I}^{\prime \prime}, M_{S}^{\prime \prime}\right\rangle .
$$

It can be shown that $C$ is pure imaginary. To do this $\left({ }^{7}\right)$, let us use (3) and (4) to calculate :

$$
\begin{aligned}
& C_{S}+C_{S}^{*}=\int \mathrm{d}^{3} k_{\mathrm{i}} u\left(\mathbf{k}_{\mathrm{i}}\right) \int \mathrm{d}^{3} k_{\mathrm{i}}^{\prime} u^{*}\left(\mathbf{k}_{\mathrm{i}}^{\prime}\right) \int \mathrm{d}^{3} k_{\mathrm{f}} \\
& {\left[\left\langle\mathrm{n}_{1}: \mathbf{k}_{\mathrm{f}} ; \mathrm{n}_{2}:-\mathbf{k}_{\mathrm{f}}\left|\left\langle\mathrm{e}_{1}: \varphi_{\mathrm{n}_{1}}\left|\left\langle\mathrm{e}_{2}: \varphi_{\mathrm{n}_{2}}|S| \mathrm{e}_{1}: \varphi_{\mathrm{n}_{1}}\right\rangle\right| \mathrm{e}_{2}: \varphi_{\mathrm{n}_{2}}\right\rangle\right| \mathrm{n}_{1}: \mathbf{k}_{\mathrm{i}} ; \mathrm{n}_{2}:-\mathbf{k}_{\mathrm{i}}\right\rangle\right.} \\
& \quad\left\langle\mathrm{n}_{1}: \mathbf{k}_{\mathrm{i}}^{\prime} ; \mathrm{n}_{2}:-\mathbf{k}_{\mathrm{i}}^{\prime}\left|\left\langle\mathrm{e}_{1}: \varphi_{\mathrm{n}_{2}}\left|\left\langle\mathrm{e}_{2}: \varphi_{\mathrm{n}_{1}}\left|S^{+}\right| \mathrm{n}_{1}: \mathbf{k}_{\mathrm{f}} ; \mathrm{n}_{2}:-\mathbf{k}_{\mathrm{f}}\right\rangle\right| \mathrm{e}_{1}: \varphi_{\mathrm{n}_{1}}\right\rangle\right| \mathrm{e}_{2}: \varphi_{\mathrm{n}_{2}}\right\rangle \\
& +\left\langle\mathrm{n}_{1}: \mathbf{k}_{\mathrm{f}} ; \mathrm{n}_{2}:-\mathbf{k}_{\mathrm{f}}\left|\left\langle\mathrm{e}_{1}: \varphi_{\mathrm{n}_{2}}\left|\left\langle\mathrm{e}_{2}: \varphi_{\mathrm{n}_{1}}|S| \mathrm{n}_{1}: \mathbf{k}_{\mathrm{i}} ; \mathrm{n}_{2}:-\mathbf{k}_{\mathrm{i}}\right\rangle\right| \mathrm{e}_{1}: \varphi_{\mathrm{n}_{1}}\right\rangle\right| \mathrm{e}_{2}: \varphi_{\mathrm{n}_{2}}\right\rangle \\
& \left.\quad\left\langle\mathrm{n}_{1}: \mathbf{k}_{\mathrm{i}}^{\prime} ; \mathrm{n}_{2}:-\mathbf{k}_{\mathrm{i}}^{\prime}\left|\left\langle\mathrm{e}_{1}: \varphi_{\mathrm{n}_{2}}\left|\left\langle\mathrm{e}_{2}: \varphi_{\mathrm{n}_{1}}\left|S^{+}\right| \mathrm{n}_{1}: \mathbf{k}_{\mathrm{f}} ; \mathrm{n}_{2}:-\mathbf{k}_{\mathrm{f}}\right\rangle\right| \mathrm{e}_{1}: \varphi_{\mathrm{n}_{2}}\right\rangle\right| \mathrm{e}_{2}: \varphi_{\mathrm{n}_{1}}\right\rangle\right] .
\end{aligned}
$$

The internal quantum numbers $m_{I}$ and $M_{S}$ play no role here, and have been omitted (writing them explicitly would amount to adding $\delta$ functions, see equations $(3 a)$ and $(3 b)$ for example). We can now use the closure relationship :

$$
\begin{aligned}
& \mathbb{1}=\int \mathrm{d}^{3} k_{\mathrm{f}}\left[\left|\mathrm{n}_{1}: \mathbf{k}_{\mathrm{f}} ; \mathrm{n}_{2}:-\mathbf{k}_{\mathrm{f}}\right\rangle\left\langle\mathrm{n}_{1}: \mathbf{k}_{\mathrm{f}} ; \mathrm{n}_{2}:-\mathbf{k}_{\mathrm{f}}\right|\right] \\
& \qquad\left[\left|\mathrm{e}_{1}: \varphi_{\mathrm{n}_{1}}\right\rangle\left|\mathrm{e}_{2}: \varphi_{\mathrm{n}_{2}}\right\rangle\left\langle\mathrm{e}_{1}: \varphi_{\mathrm{n}_{1}}\left|\left\langle\mathrm{e}_{2}: \varphi_{\mathrm{n}_{2}}|+| \mathrm{e}_{1}: \varphi_{\mathrm{n}_{2}}\right\rangle\right| \mathrm{e}_{2}: \varphi_{\mathrm{n}_{1}}\right\rangle\left\langle\mathrm{e}_{1}: \varphi_{\mathrm{n}_{2}}\right|\left\langle\mathrm{e}_{2}: \varphi_{\mathrm{n}_{1}}\right|\right]
\end{aligned}
$$

and the unitarity of the operator $S$ to write :

$$
\begin{aligned}
& C_{S}+C_{S}^{*}=\int \mathrm{d}^{3} k_{\mathrm{i}} u\left(\mathbf{k}_{\mathrm{i}}\right) \int \mathrm{d}^{3} k_{\mathrm{i}}^{\prime} u^{*}\left(\mathbf{k}_{\mathrm{i}}^{\prime}\right) \\
& \quad\left[\left\langle\mathrm{n}_{1}: \mathbf{k}_{\mathrm{i}}^{\prime} ; \mathrm{n}_{2}:-\mathbf{k}_{\mathrm{i}}^{\prime}\left|\left\langle\mathrm{e}_{1}: \varphi_{\mathrm{n}_{2}}\left|\left\langle\mathrm{e}_{2}: \varphi_{\mathrm{n}_{1}}\right|\right]\left[\left|\mathrm{n}_{1}: \mathbf{k}_{\mathrm{i}} ; \mathrm{n}_{2}:-\mathbf{k}_{\mathrm{i}}\right\rangle\left|\mathrm{e}_{1}: \varphi_{\mathrm{n}_{1}}\right\rangle\left|\mathrm{e}_{2}: \varphi_{\mathrm{n}_{2}}\right\rangle\right] .\right.\right.\right.\right.
\end{aligned}
$$

On the right hand side of (22), one recognizes the scalar product of two different initial states : in one of the states, $e_{1}$ is bound to $n_{1}$ and $e_{2}$ to $n_{2}$, in the other the opposite situation occurs. Since in the initial state the nuclei are very far apart, the overlapping of the wave function of $e_{1}$ and $e_{2}$ is zero, and one then obtains :

$$
C_{S}+C_{S}^{*}=0 \text {. }
$$

We can therefore set :

$$
C_{S}=i A_{\mathrm{exch}}^{S}
$$

where $C_{S}$ is defined by $(20)$ and $A_{\text {exch. }}$ is a real coefficient $\left({ }^{8}\right)$.

( 7$)$ In the Appendix, another demonstration using the phase shift method is given

$\left({ }^{8}\right)$ The Schwarz inequality gives :

$$
\left|C_{S}\right|^{2}=\left|A_{S}\right|^{2} \leqslant A_{\mathrm{d}} A_{\mathrm{t}}=A_{\mathrm{t}}\left(1-A_{\mathrm{t}}\right) \leqslant \frac{1}{4}
$$


If $v_{I}$ denotes the identity operator in the I variable space, one can write :

$$
\begin{aligned}
\left\langle m_{I}, M_{S}\left|\rho_{1} \times\left(\mathbb{1}_{I} \otimes \operatorname{Tr}_{I}\left\{\rho_{2}\right\}\right)\right| m_{I}^{\prime},\right. & \left.M_{S}^{\prime}\right\rangle \\
= & \sum_{m_{I}^{\prime \prime}} \sum_{M_{S}^{\prime \prime}}\left\langle m_{I}, M_{S}\left|\rho_{1}\right| m_{I}^{\prime \prime \prime} M_{S}^{\prime \prime}\right\rangle \delta_{m_{I}^{\prime} m_{I}^{\prime \prime}} \\
& \sum_{m_{I}^{\prime \prime}}\left\langle m_{I}^{\prime \prime}, M_{S}^{\prime \prime}\left|\rho_{2}\right| m_{I}^{\prime \prime}, M_{S}^{\prime}\right\rangle \\
= & \sum_{m_{I}^{\prime \prime}} \sum_{M_{S}^{\prime \prime}}\left\langle m_{I}, M_{S}\left|\rho_{1}\right| m_{I}^{\prime}, M_{S}^{\prime \prime}\right\rangle\left\langle m_{I}^{\prime \prime}, M_{S}^{\prime \prime}\left|\rho_{2}\right| m_{I}^{\prime \prime}, M_{S}^{\prime}\right\rangle .
\end{aligned}
$$

In the same way:

$$
\left\langle m_{I}, M_{S}\left|\left(\mathbb{1}_{I} \otimes \operatorname{Tr}_{I}\left\{\rho_{2}\right\}\right) \times \rho_{1}\right| m_{I}^{\prime}, M_{S}^{\prime}\right\rangle=\sum_{m_{I}^{\prime \prime}} \sum_{M_{S}^{\prime \prime}}\left\langle m_{I}^{\prime \prime}, M_{S}\left|\rho_{2}\right| m_{I}^{\prime \prime}, M_{S}^{\prime \prime}\right\rangle\left\langle m_{I}, M_{S}^{\prime \prime}\left|\rho_{1}\right| m_{I}^{\prime}, M_{S}^{\prime}\right\rangle .
$$

These equalities allow us to write the terms in $A_{\text {exch. }}^{S}$ in an operatorial form, and we obtain for the density operator $\rho_{\mathrm{f}}\left(\mathrm{n}_{1}, \mathrm{e}\right)$ after collision :

$$
\rho_{\mathrm{f}}\left(\mathrm{n}_{1}, \mathrm{e}\right)=\rho_{1}+A_{\mathrm{t}}\left(\operatorname{Tr}_{S}\left\{\rho_{1}\right\} \otimes \operatorname{Tr}_{I}\left\{\rho_{2}\right\}-\rho_{1}\right)-i A_{\text {exch. }}^{S}\left[\rho_{1}, \nabla_{I} \otimes \operatorname{Tr}_{I}\left\{\rho_{2}\right\}\right] .
$$

In a similar way, the density operator of the atom labeled by $\mathrm{n}_{2}$ is, after collision :

$$
\rho_{\mathrm{f}}\left(\mathrm{n}_{2}, \mathrm{e}\right)=\rho_{2}+A_{\mathrm{t}}\left(\operatorname{Tr}_{S}\left\{\rho_{2}\right\} \otimes \operatorname{Tr}_{I}\left\{\rho_{1}\right\}-\rho_{2}\right)-i A_{\text {exch. }}^{\mathrm{S}}\left[\rho_{2}, \mathbb{1}_{I} \otimes \operatorname{Tr}_{I}\left\{\rho_{1}\right\}\right]
$$

2.2.2 Physical interpretation. - Equation (26) describes the evolution of the atomic internal variables when the indistinguishability between electrons is taken into account. The equation analoguous to (16) is now :

where :

$$
\frac{\mathrm{d}}{\mathrm{d} t} \rho\left(\text { atom 1) }\left.\right|_{\text {coll. }}=\frac{1}{T_{\text {coll. }}}\left\{-\rho_{1}+\operatorname{Tr}_{S}\left\{\rho_{1}\right\} \otimes \operatorname{Tr}_{I}\left\{\rho_{2}\right\}+i \kappa_{S}\left[\vartheta_{I} \otimes \operatorname{Tr}_{I}\left\{\rho_{2}\right\}, \rho_{1}\right]\right\}\right.
$$

$$
\kappa_{\mathrm{S}}=\frac{A_{\mathrm{exch}}^{\mathrm{S}}}{A_{\mathrm{t}}}
$$

(this coefficient is noted $\kappa$ in Ref. [9]). One immediately notices that the first two terms of the right hand side of (27) are nothing but the terms which already appeared in (16) : therefore, the electron identity does not affect the way electronic polarization is transferred from $n_{1}$-type atoms to $n_{2}$-type atoms.

The only effect of the Pauli principle is to add to the right hand side of (27) a term proportional to $\kappa_{S}$ [that is to the coefficient $C_{S}$ defined in (20)]. It can be seen on this expression that the new term arises from interference effects between direct and transfer scattering processes in the same final direction $\mathbf{k}_{\mathbf{f}}$. Since this term appears as a commutator, it does not change the trace of the density operator; the introduction of the Pauli principle is equivalent to an effective hamiltonian :

$$
H_{\text {coll. }}=-\frac{\hbar \kappa_{S}}{T_{\text {coll. }}} 0_{I} \otimes \operatorname{Tr}_{I}\left\{\rho_{2}\right\} .
$$

This hamiltonian acts on the electronic spin variables of atoms 1 and depends only on the electronic spin density operator of atoms 2 .

If :

$$
\operatorname{Tr}_{I}\left\{\rho_{2}\right\} \propto \mathbb{1}_{S}
$$

(rotation invariant electronic density matrix for atoms 2), the Pauli principle term in (27) vanishes. More generally, since electrons are spin $1 / 2$ particles, we can write :

$$
\operatorname{Tr}_{I}\left\{\rho_{2}\right\}=\frac{1}{2}\left[0_{S}+\langle\sigma\rangle_{2} \cdot \sigma\right]
$$

where :

$$
\mathbf{S}=\frac{\hbar}{2} \boldsymbol{\sigma}
$$


and :

$$
\langle\sigma\rangle_{2}=\operatorname{Tr}_{S}\left\{\sigma \operatorname{Tr}_{I}\left\{\rho_{2}\right\}\right\} .
$$

The Pauli principle term in (27) then takes the form :

$$
i \frac{\kappa_{S}}{2 T_{\text {coll. }}}\left[\langle\sigma\rangle_{2} \cdot \sigma, \rho_{1}\right] .
$$

In other words, spin exchange collisions involve an apparent magnetic field acting on the electronic spin of atoms $1\left({ }^{9}\right)$. This apparent field is parallel and proportional to the electron spin polarization of species 2 . The fact that only the electronic orientation of each species affects the density matrix evolution of the other has been pointed out by F. Grossetête [18] and P. Valberg [19] (see also [2]).

It is well known that indistinguishability terms relative to identical particles vanish when the particles are in orthogonal spin states and when their spins remain unaffected during the physical processes under study (see for example the discussion in chapter XIV, $\S \mathrm{D}-2-\mathrm{b}-\beta$ of Ref. [17]). Physically, this arises because, in principle, the spin states could be used to distinguish the two particles. Let us assume that the internal electronic and nuclear variables of atom 1 are not correlated, that is :

$$
\rho_{1}=\rho_{1}^{I} \otimes \rho_{1}^{S} .
$$

The electron spin exchange shift term then becomes :

where :

$$
i \kappa_{S} \rho_{1}^{I} \otimes\left[\rho_{2}^{S}, \rho_{1}^{S}\right],
$$

$$
\rho_{2}^{S}=\operatorname{Tr}_{I}\left\{\rho_{2}\right\} .
$$

A necessary and sufficient condition for the two electrons to be in orthogonal spin states is that the density matrices are such that :

$$
\rho_{1}^{S} \rho_{2}^{S}=\rho_{2}^{S} \rho_{1}^{S}=0 .
$$

This is obviously true if $\rho_{1}^{S}$ and $\rho_{2}^{S}$ are projectors onto two orthogonal states. Conversely, if condition (35) is fulfilled, $\rho_{1}^{S}$ and $\rho_{2}^{S}$ can be diagonalized in the same basis. If their product is zero, the matrices in this basis never have non-zero populations in the same level and the electron spins are in orthogonal spin states. Now, it is easy to see that condition (35) implies that the spin exchange shift term (33) vanishes, and we check in this way that particle indistinguishability effects then disappear.

In fact, one notices immediately on (33) that condition (35) is too strong for the Pauli principle term to disappear : it is sufficient that $\rho_{1}^{S}$ and $\rho_{2}^{s}$ commute with each other. It must nevertheless be kept in mind that this condition is only valid because we chose in (32) a $\rho_{1}$ density matrix without correlations between the spins. In general, when $\mathbf{I}$ and $\mathbf{S}$ are correlated, spin exchange shift terms can subsist even if $\rho_{1}^{S}=\rho_{2}^{S}$ (see $\S 3$, where spin exchange shift terms are calculated when $\rho_{1}=\rho_{2}$ ).

2.3 INDISTINGUISHABLE NUCLEI AND ELECTRONS. - We shall assume in this section that the two nuclei $n_{1}$ and $\mathrm{n}_{2}$ are also indistinguishable particles. For example, the collision occurs between two hydrogen atoms, so that $\mathrm{n}_{1}$ and $\mathrm{n}_{2}$ are two protons (spin $1 / 2$ particles). The notation $P_{\mathrm{n}}$ will be used for the exchange operator between $n_{1}$ and $n_{2}$. This operator acts only in the nuclear variable state space, and :

$$
\begin{aligned}
P_{\mathrm{n}}\left|\mathrm{n}_{1}: \mathbf{k} ; \mathrm{n}_{2}:-\mathbf{k}\right\rangle\left|\mathrm{n}_{1}: m_{I}\right\rangle\left|\mathrm{n}_{2}: m_{I}^{\prime}\right\rangle\left|\mathrm{e}_{1}: \varphi_{\mathrm{n}_{1}}\right\rangle\left|\mathrm{e}_{2}: \varphi_{\mathrm{n}_{2}}\right\rangle \\
\quad=\left|\mathrm{n}_{1}:-\mathbf{k} ; \mathrm{n}_{2}: \mathbf{k}\right\rangle\left|\mathrm{n}_{1}: m_{I}^{\prime}\right\rangle\left|\mathrm{n}_{2}: m_{I}\right\rangle\left|\mathrm{e}_{1}: \varphi_{\mathrm{n}_{2}}\right\rangle\left|\mathrm{e}_{2}: \varphi_{\mathrm{n}_{1}}\right\rangle
\end{aligned}
$$

(it must be kept in mind that states like $\left|\mathrm{e}_{1}: \varphi_{\mathrm{n}_{1}}\right\rangle$ are not invariant under the action of $P_{\mathrm{n}}$ ).

2.3.1 Density operator after collision. - Before the collision occurs, the two atom density operator is $\left({ }^{10}\right)$ :

$$
\frac{1}{2}\left[1-P_{\mathrm{n}}\right]\left[1-P_{\mathrm{e}}\right] \sigma_{\text {init. }} \frac{1}{2}\left[1-P_{\mathrm{n}}\right]\left[1-P_{\mathrm{e}}\right]
$$

\footnotetext{
$\left({ }^{9}\right)$ Hence the notation $-\boldsymbol{\mu} . \delta \mathbf{H}$ for $H_{\text {coll. }}$ in equation (VI.8) of Ref. [2].

$\left({ }^{10}\right)$ We assume here that the nuclei are fermions. When they are bosons, it is sufficient, in all the following calculations, to change the sign of all terms linear in $P_{\mathrm{n}}$ [terms in $C_{I}$ and $D_{I S}$ ].
} 
where $\sigma_{\text {init. }}$ is still given by (1). Except for the fact that two exchange operators $P$ now appear, the calculation is very similar to the one of section 2.2. Equation (19) has to be replaced by :

$$
\begin{aligned}
& \left\langle m_{I}, M_{S}\left|\rho_{\mathrm{f}}(\mathrm{n}, \mathrm{e})\right| m_{I}^{\prime}, M_{S}^{\prime}\right\rangle= \\
& \frac{1}{2} \int \mathrm{d}^{3} k_{\mathrm{i}} u\left(\mathbf{k}_{\mathrm{i}}\right) \int \mathrm{d}^{3} k_{\mathrm{i}}^{\prime} u^{*}\left(\mathbf{k}_{\mathrm{i}}^{\prime}\right) \int \mathrm{d}^{3} k_{\mathrm{f}} \sum_{m_{I}^{\prime \prime}} \sum_{M_{S}^{\prime \prime}} \\
& {\left[\left\langle\mathrm{n}_{1}: \mathbf{k}_{\mathrm{f}} ; \mathrm{n}_{2}:-\mathbf{k}_{\mathrm{f}}\right|\left\langle\mathrm{n}_{1}: m_{I}\left|<\mathrm{n}_{2}: m_{I}^{\prime \prime}\right|<\mathrm{e}_{1}: \varphi_{\mathrm{n}_{1}}, M_{S}\left|<\mathrm{e}_{2}: \varphi_{\mathrm{n}_{2}}, M_{S}^{\prime \prime}\right|\right]\right.} \\
& {\left[1-P_{\mathrm{n}}\right]\left[1-P_{\mathrm{e}}\right] S \sigma_{\text {init. }} S^{+}\left[1-P_{\mathrm{n}}\right]\left[1-P_{\mathrm{e}}\right]} \\
& {\left[\left|\mathrm{n}_{1}: \mathbf{k}_{\mathrm{f}} ; \mathrm{n}_{2}:-\mathbf{k}_{\mathrm{f}}\right\rangle\left|\mathrm{n}_{1}: m_{I}^{\prime}\right\rangle\left|\mathrm{n}_{2}: m_{I}^{\prime \prime}\right\rangle\left|\mathrm{e}_{1}: \varphi_{\mathrm{n}_{1}}, M_{S}^{\prime}\right\rangle\left|\mathrm{e}_{2}: \varphi_{\mathrm{n}_{2}}, M_{S}^{\prime \prime}\right\rangle\right] \text {. }}
\end{aligned}
$$

To obtain this equality, we have made use of the fact that $S$ commutes with both operators $P_{\mathrm{e}}$ and $P_{\mathrm{n}}$, and of the relationship $P_{\mathrm{e}}^{2}=P_{\mathrm{n}}^{2}=1$. The factor $1 / 2$ which stands in front of the right hand side of (36) is necessary if the integral over $\mathbf{k}_{\mathbf{f}}$ is taken over all $\mathbf{k}_{\mathrm{f}}$ directions ( $4 \pi$ steradians). This is because the action of the operator

$$
\left[1-P_{\mathrm{n}}\right]\left[1-P_{\mathrm{e}}\right]
$$

on the two kets

$$
\left|\mathrm{n}_{1}= \pm \mathbf{k}_{\mathrm{f}} ; \mathrm{n}_{2}=\mp \mathbf{k}_{\mathrm{f}}\right\rangle\left|\mathrm{e}_{1}: \varphi_{\mathrm{n}_{1}}\right\rangle\left|\mathrm{e}_{2}: \varphi_{\mathrm{n}_{2}}\right\rangle
$$

(we omit all spin variables which play no role here) gives the same ket. Therefore, in order to avoid counting twice the same physical final states, either the $k_{\mathrm{f}}$ integral in (36) must be restricted to a half space, or a factor $1 / 2$ must be introduced as we have done.

When the two products $\left[1-P_{n}\right]\left[1-P_{e}\right]$ are expanded in (36), the number of terms which appears is 16 . Since we now discuss nuclear exchange effects, it is convenient to classify these terms according to the number of operators $P_{\mathrm{n}}$ involved. The first four terms contain no such operator at all and are actually nothing but the four terms already discussed in the preceding section (multiplied by a factor $1 / 2$ ). Their contribution is therefore simply given by $(26 a)$. There are now four terms which include one $P_{\mathrm{n}}$ operator on both sides of $S \rho_{\text {init. }} S^{+}$. Calculating these terms amounts to doing the same calculations as before but after permutation of $n_{1}$ and $n_{2}$ in the final state. In other words, these four terms merely correspond to the calculation given in section 2.2 for the density operator of atom $\mathrm{n}_{2}$ after collision. Equation $(26 b)$ therefore gives their contribution directly.

The remaining 8 terms have not been considered before and represent the effect of nuclear indistinguishability. Each of these terms contains one operator $P_{\mathrm{n}}$ in the right or in the left of $S \sigma_{\text {init. }} S^{+}$. We shall classify the terms according to the number of operators $P_{\mathrm{e}}$ included. The first two terms have no operator $P_{\mathrm{e}}$ at all (pure nuclear exchange) and include $-P_{\mathrm{n}} S \rho_{\text {init. }} S^{+}$or $-S \rho_{\text {init. }} S^{+} P_{\mathrm{n}}$.

The contribution of the latter for example to (36) is :

$$
\begin{gathered}
-\frac{1}{2} \mathrm{~d}^{3} k_{\mathrm{i}} u\left(\mathbf{k}_{\mathrm{i}}\right) \int \mathrm{d}^{3} k_{\mathrm{i}}^{\prime} u^{*}\left(\mathbf{k}_{\mathrm{i}}^{\prime}\right) \int \mathrm{d}^{3} k_{\mathrm{f}} \sum_{m_{I}^{\prime \prime}} \sum_{M_{S}^{\prime \prime}} \\
S_{\mathrm{d}}\left(\mathbf{k}_{\mathrm{f}}, \mathbf{k}_{\mathrm{i}}\right) S_{\mathrm{t}}^{*}\left(-\mathbf{k}_{\mathrm{f}}, \mathbf{k}_{\mathrm{i}}^{\prime}\right) \\
\left\langle m_{I} M_{S}\left|\rho_{1}\right| m_{I}^{\prime \prime} M_{S}^{\prime}\right\rangle\left\langle m_{I}^{\prime \prime} M_{S}^{\prime \prime}\left|\rho_{2}\right| m_{I}^{\prime} M_{S}^{\prime \prime}\right\rangle .
\end{gathered}
$$

If we set :

$$
C_{I}=\int \mathrm{d}^{3} k_{\mathrm{i}} u\left(\mathbf{k}_{\mathrm{i}}\right) \int \mathrm{d}^{3} k_{\mathrm{i}}^{\prime} u^{*}\left(\mathbf{k}_{\mathrm{i}}^{\prime}\right) \int \mathrm{d}^{3} k_{\mathrm{f}} S_{\mathrm{d}}\left(\mathbf{k}_{\mathrm{f}}, \mathbf{k}_{\mathrm{i}}\right) S_{\mathrm{t}}^{*}\left(-\mathbf{k}_{\mathrm{f}}, \mathbf{k}_{\mathrm{i}}^{\prime}\right)
$$

(we shall see below that $C_{I}$, like $C_{S}$, is pure imaginary), we can write this contribution :

$$
-\frac{1}{2} C_{I}\left\langle m_{I} M_{S}\left|\rho_{1} \times\left(\operatorname{Tr}_{S}\left\{\rho_{2}\right\} \otimes \mathbb{1}_{S}\right)\right| m_{I}^{\prime} M_{S}^{\prime}\right\rangle .
$$

In the same way, the term in $-P_{\mathrm{n}} S \sigma_{\text {init. }} S^{+}$takes the form :

$$
-\frac{1}{2} C_{I}^{*}\left\langle m_{I} M_{S}\left|\left(\operatorname{Tr}_{S}\left\{\rho_{2}\right\} \otimes \mathbb{1}_{S}\right) \times \rho_{1}\right| m_{I}^{\prime} M_{S}^{\prime}\right\rangle .
$$


Let us now consider the two terms in $P_{\mathrm{e}} P_{\mathrm{n}} S \sigma_{\text {init. }} S^{+} P_{\mathrm{e}}$ and $P_{\mathrm{e}} S \sigma_{\text {init. }} S^{+} P_{\mathrm{e}} P_{\mathrm{n}}$ (two operators $P_{\mathrm{e}}$ included). The calculation is of the same type as previously, and one obtains for the first term :

$$
\begin{aligned}
-\frac{1}{2} \int \mathrm{d}^{3} k_{\mathrm{i}} u\left(\mathbf{k}_{\mathrm{i}}\right) \int \mathrm{d}^{3} k_{\mathrm{i}}^{\prime} u^{*}\left(\mathbf{k}_{\mathrm{i}}^{\prime}\right) \int \mathrm{d}^{3} k_{\mathrm{f}} \sum_{m_{I}^{\prime \prime}} \sum_{M_{S}^{\prime \prime}} S_{\mathrm{d}}\left(-\mathbf{k}_{\mathrm{f}}, \mathbf{k}_{\mathrm{i}}\right) S_{\mathrm{I}}^{*}\left(\mathbf{k}_{\mathrm{f}}, \mathbf{k}_{\mathrm{i}}^{\prime}\right) \\
\left\langle m_{I}^{\prime \prime} M_{S}^{\prime \prime}\left|\rho_{1}\right| m_{I}^{\prime} M_{S}^{\prime \prime}\right\rangle\left\langle m_{I} M_{S}\left|\rho_{2}\right| m_{I}^{\prime \prime} M_{S}^{\prime}\right\rangle \\
=-\frac{1}{2} C_{I}\left\langle m_{I} M_{S}\left|\rho_{2} \times\left(\operatorname{Tr}_{S}\left\{\rho_{1}\right\} \otimes \mathbb{v}_{S}\right)\right| m_{I}^{\prime} M_{S}^{\prime}\right\rangle,
\end{aligned}
$$

and for the second :

$$
-\frac{1}{2} C_{I}^{*}\left\langle m_{I} M_{S}\left|\left(\operatorname{Tr}_{S}\left\{\rho_{1}\right\} \otimes \mathbb{1}_{S}\right) \times \rho_{2}\right| m_{I}^{\prime} M_{S}^{\prime}\right\rangle .
$$

There are now only four terms left to evaluate. Two of them are in $P_{\mathrm{e}} P_{\mathrm{n}} S \sigma_{\text {init. }} S^{+}$and $S \sigma_{\text {init }} S^{+} P_{\mathrm{e}} P_{\mathrm{n}}$ respectively. The latter for example gives a contribution :

$$
\begin{aligned}
\frac{1}{2} \int \mathrm{d}^{3} k_{\mathrm{i}} u\left(\mathbf{k}_{\mathrm{i}}\right) \int \mathrm{d}^{3} k_{\mathrm{i}}^{\prime} u^{*}\left(\mathbf{k}_{\mathrm{i}}^{\prime}\right) \int \mathrm{d}^{3} k_{\mathrm{f}} \sum_{m_{I}^{\prime \prime}} \sum_{M_{S}^{\prime \prime}} S_{\mathrm{d}}\left(\mathbf{k}_{\mathrm{f}}, \mathbf{k}_{\mathrm{i}}\right) S_{\mathrm{d}}^{*}\left(-\mathbf{k}_{\mathrm{f}}, \mathbf{k}_{\mathrm{i}}^{\prime}\right) \\
\\
\left\langle m_{I} M_{S}\left|\rho_{1}\right| m_{I}^{\prime \prime} M_{S}^{\prime \prime}\right\rangle\left\langle m_{I}^{\prime \prime} M_{S}^{\prime \prime}\left|\rho_{2}\right| m_{I}^{\prime} M_{S}^{\prime}\right\rangle=\frac{1}{2} D_{I S}\left\langle m_{I} M_{S}\left|\rho_{1} \times \rho_{2}\right| m_{I}^{\prime} M_{S}^{\prime}\right\rangle .
\end{aligned}
$$

The coefficient $D_{I S}$ is defined by :

$$
D_{I S}=\int \mathrm{d}^{3} k_{\mathrm{i}} u\left(\mathbf{k}_{\mathrm{i}}\right) \int \mathrm{d}^{3} k_{\mathrm{i}}^{\prime} u^{*}\left(\mathbf{k}_{\mathrm{i}}^{\prime}\right) \int \mathrm{d}^{3} k_{\mathrm{f}} S_{\mathrm{d}}\left(\mathbf{k}_{\mathrm{f}}, \mathbf{k}_{\mathrm{i}}\right) S_{\mathrm{d}}^{*}\left(-\mathbf{k}_{\mathrm{f}}, \mathbf{k}_{\mathrm{i}}^{\prime}\right) .
$$

The term in $P_{\mathrm{e}} P_{\mathrm{n}} S \sigma_{\text {init. }} S^{+}$is the hermitian conjugate of this result and is therefore equal to :

$$
\frac{1}{2} D_{I S}^{*}\left\langle m_{I} M_{S}\left|\rho_{2} \times \rho_{1}\right| m_{I}^{\prime} M_{S}^{\prime}\right\rangle .
$$

As for the last two terms, in $P_{\mathrm{e}} S \sigma_{\text {init. }} S^{+} P_{\mathrm{n}}$ and $P_{\mathrm{n}} S \sigma_{\text {init. }} S^{+} P_{\mathrm{e}}$, they can also be calculated in the same way. The former, for example, is found equal to :

$$
\frac{1}{2} E_{I S}\left\langle m_{I} M_{S}\left|\operatorname{Tr}_{2}\left\{P_{\mathrm{e}} \rho_{1} \otimes \rho_{2} P_{\mathrm{n}}\right\}\right| m_{I}^{\prime} M_{S}^{\prime}\right\rangle .
$$

In this expression, $\operatorname{Tr}_{2}=\operatorname{Tr}_{I_{2}} \operatorname{Tr}_{S_{2}}$ is the partial trace over all internal variables of atom 2, and $E_{I S}$ is the coefficient :

$$
E_{I S}=\int \mathrm{d}^{3} k_{\mathrm{i}} u\left(\mathbf{k}_{\mathrm{i}}\right) \int \mathrm{d}^{3} k_{\mathrm{i}}^{\prime} u^{*}\left(\mathbf{k}_{\mathrm{i}}^{\prime}\right) \int \mathrm{d}^{3} k_{\mathrm{f}} S_{\mathrm{t}}\left(\mathbf{k}_{\mathrm{f}}, \mathbf{k}_{\mathrm{i}}\right) S_{\mathrm{t}}^{*}\left(-\mathbf{k}_{\mathrm{f}}, \mathbf{k}_{\mathrm{i}}^{\prime}\right)
$$

Before we discuss the physical interpretation of all the different terms which have been found, it is convenient to remark that :

$$
C_{I}+C_{I}^{*}=0
$$

$\left(C_{I}\right.$ is pure imaginary) and :

$$
D_{I S}+E_{I S}=0 .
$$

The proof of these two equalities is similar to the proof of (22). Using the same argument (and interchanging particles $\mathrm{n}$ and e when necessary; see $(4 b, c))$ :

$$
\begin{aligned}
& C_{I}+C_{I}^{*}=\int \mathrm{d}^{3} k_{\mathrm{i}} u\left(\mathbf{k}_{\mathrm{i}}\right) \int \mathrm{d}^{3} k_{\mathrm{i}}^{\prime} u^{*}\left(\mathbf{k}_{\mathrm{i}}^{\prime}\right) \\
& \quad\left[\left\langle\mathrm{n}_{1}: \mathbf{k}_{\mathrm{i}}^{\prime} ; \mathrm{n}_{2}:-\mathbf{k}_{\mathrm{i}}^{\prime}\left|\left\langle\mathrm{e}_{1}: \varphi_{\mathrm{n}_{2}}\left|\left\langle\mathrm{e}_{2}: \varphi_{\mathrm{n}_{1}}\right|\right] S^{+} S\left[\left|\mathrm{n}_{1}:-\mathbf{k}_{\mathrm{i}} ; \mathrm{n}_{2}: \mathbf{k}_{\mathrm{i}}\right\rangle\left|\mathrm{e}_{1}: \varphi_{\mathrm{n}_{1}}\right\rangle\left|\mathrm{e}_{2}: \varphi_{\mathrm{n}_{2}}\right\rangle\right] .\right.\right.\right.\right.
\end{aligned}
$$


Since $S^{+} S=1, C_{I}+C_{I}^{*}$ is the scalar product of two kets for which each of the nuclei moves in opposite directions and each electron is bound to different nuclei. The integration over nuclear position variables then gives zero and (41) is established. In the same way, one obtains :

$D_{I S}+E_{I S}=\int \mathrm{d}^{3} k_{\mathrm{i}} u\left(\mathbf{k}_{\mathrm{i}}\right) \int \mathrm{d}^{3} k_{\mathrm{i}}^{\prime} u^{*}\left(\mathbf{k}_{\mathrm{i}}^{\prime}\right)$

$\left[\left\langle\mathrm{n}_{1}: \mathbf{k}_{\mathrm{i}}^{\prime} ; \mathrm{n}_{2}:-\mathbf{k}_{\mathrm{i}}^{\prime}\left|\left\langle\mathrm{e}_{1}: \varphi_{\mathrm{n}_{1}}\left|\left\langle\mathrm{e}_{2}: \varphi_{\mathrm{n}_{2}}\right|\right] S^{+} S\left[\left|\mathrm{n}_{1}:-\mathbf{k}_{\mathrm{i}} ; \mathrm{n}_{2}:+\mathbf{k}_{\mathrm{i}}\right\rangle\left|\mathrm{e}_{1}: \varphi_{\mathrm{n}_{1}}\right\rangle\left|\mathrm{e}_{2}: \varphi_{\mathrm{n}_{2}}\right\rangle\right]=0\right.\right.\right.\right.$.

This expression vanishes because the nuclear wave packets of the bra and the ket do not overlap.

It is also easy to see on definitions $(40 a)$ and $(40 b)$ that $D_{I S}$ and $E_{I S}$ are real coefficients.

To summarize, the expansion :

\begin{tabular}{ccc}
{$\left[1-P_{\mathrm{e}}\right]\left[1-P_{\mathrm{n}}\right]$} & $\times$ & {$\left[1-P_{\mathrm{e}}\right]\left[1-P_{\mathrm{n}}\right]$} \\
& $=$ & \\
\hline$+P_{\mathrm{n}}\left[1-P_{\mathrm{e}}\right]$ & $\times$ & $P_{\mathrm{n}}\left[1-P_{\mathrm{e}}\right]$ \\
\hline$-P_{\mathrm{n}}$ & $\times$ & 1 \\
-1 & $\times$ & $P_{\mathrm{n}}$ \\
$-P_{\mathrm{e}} P_{\mathrm{n}}$ & $\times$ & $P_{\mathrm{e}}$ \\
$-P_{\mathrm{e}}$ & $\times$ & $P_{\mathrm{e}} P_{\mathrm{n}}$ \\
\hline$+P_{\mathrm{e}} P_{\mathrm{n}}$ & $\times$ & 1 \\
+1 & $\times$ & $P_{\mathrm{e}} P_{\mathrm{n}}$ \\
$+P_{\mathrm{e}}$ & $\times$ & $P_{\mathrm{n}}$ \\
$+P_{\mathrm{n}}$ & $\times$ & $P_{\mathrm{e}}$
\end{tabular}

corresponds for the internal variable density operator after collision to :

$$
\begin{aligned}
& \rho_{\mathrm{f}}(\mathrm{e}, \mathrm{n})= \\
& \frac{1}{2}\left(1-A_{\mathrm{t}}\right) \rho_{1}+\frac{A_{\mathrm{t}}}{2} \operatorname{Tr}_{S}\left\{\rho_{1}\right\} \otimes \operatorname{Tr}_{I}\left\{\rho_{2}\right\}-i \frac{A_{\mathrm{exch}}^{S}}{2}\left[\rho_{1}, \mathbb{1}_{I} \otimes \operatorname{Tr}_{I}\left\{\rho_{2}\right\}\right] \\
& +\frac{1}{2}\left(1-A_{\mathrm{t}}\right) \rho_{2}+\frac{A_{\mathrm{t}}}{2} \operatorname{Tr}_{S}\left\{\rho_{2}\right\} \otimes \operatorname{Tr}_{I}\left\{\rho_{1}\right\}-i \frac{A_{\mathrm{exch}}^{S}\left[\rho_{2}, \mathbb{1}_{I} \otimes \operatorname{Tr}_{I}\left\{\rho_{1}\right\}\right]}{2} \\
& -\frac{i}{2} A_{\mathrm{exch} .}^{I}\left[\rho_{1}, \operatorname{Tr}_{S}\left\{\rho_{2}\right\} \otimes \mathbb{1}_{S}\right]-\frac{i}{2} A_{\mathrm{exch}}^{I}\left[\rho_{2}, \operatorname{Tr}_{S}\left\{\rho_{1}\right\} \otimes \vartheta_{S}\right] \\
& -\frac{1}{2} E_{I S}\left(\rho_{1} \times \rho_{2}+\rho_{2} \times \rho_{1}-\operatorname{Tr}_{2}\left\{P_{\mathrm{n}} \rho_{1}(1) \otimes \rho_{2}(2) P_{\mathrm{e}}+P_{\mathrm{e}} \rho_{1}(1) \otimes \rho_{2}(2) P_{\mathrm{n}}\right\}\right)
\end{aligned}
$$

Here we have defined the real coefficient $A_{\text {exch. by : }}^{I}$

$$
i A_{\text {exch. }}^{I}=C_{I}
$$

2.3.2 Physical interpretation. - It can be first noted that, in equation (45), the two density operators $\rho_{1}$ and $\rho_{2}$ play a perfectly symmetric role, as can be expected when $n_{1}$ and $n_{2}$ are identical particles. One can also see that the only terms which contribute to the trace of $\rho_{\mathrm{f}}(\mathrm{e}, \mathrm{n})$ are the terms in $A_{\mathrm{t}}$ or $\left(1-A_{\mathrm{t}}\right)$, that is the terms 
which were discussed in section 1.1 (distinguishable particles). All commutators have a zero trace, so the only terms which do not obviously have a zero trace are the terms in $E_{I S}$. Nevertheless, since :

$$
\begin{aligned}
\operatorname{Tr}\left\{P_{\mathrm{e}} \rho_{1} \otimes \rho_{2} P_{\mathrm{n}}\right\} & \\
& =\sum_{M_{S} M_{S}^{\prime}} \sum_{m_{I} m_{I}^{\prime}}\left\langle 1: m_{I}, M_{S} ; 2: m_{I}^{\prime}, M_{S}^{\prime}\left|P_{\mathrm{e}} \rho_{1} \otimes \rho_{2} P_{\mathrm{n}}\right| 1: m_{I}, M_{S} ; 2: m_{I}^{\prime}, M_{S}^{\prime}\right\rangle \\
& =\sum_{M_{S} M_{S}^{\prime}} \sum_{m_{I} m_{I}^{\prime}}\left\langle m_{I}, M_{S}^{\prime}\left|\rho_{1}\right| m_{I}^{\prime}, M_{S}\right\rangle\left\langle m_{I}^{\prime}, M_{S}\left|\rho_{2}\right| m_{I}, M_{S}^{\prime}\right\rangle \\
& =\sum_{m_{I} M_{S}^{\prime}}\left\langle m_{I}, M_{S}^{\prime}\left|\rho_{1} \times \rho_{2}\right| m_{I}, M_{S}^{\prime}\right\rangle \\
& =\operatorname{Tr}\left\{\rho_{1} \times \rho_{2}\right\},
\end{aligned}
$$

it can easily be shown that none of the indistinguishability terms changes the trace of $\rho_{\mathrm{f}}(\mathrm{e}, \mathrm{n})$.

Nevertheless, they do in general change the partial traces of this operator over either $\mathbf{I}$ or $\mathbf{S}$ variables. The effect of the terms proportional to $A_{\text {exch. }}^{S}$, already discussed, is a rotation of the electronic spin variables around $\langle\mathbf{S}\rangle_{1}$ or $\langle\mathbf{S}\rangle_{2}$ (electronic spin orientations associated to $\rho_{1}$ and $\rho_{2}$ ). The terms proportional to $A_{\text {exch. }}^{I}$ are similar, but the effective hamiltonian now acts only on the nuclear variables. This is not surprising since these terms arise from the nucleus identity (no $P_{\mathrm{e}}$ operator involved). If either matrix $\rho_{1}$ or matrix $\rho_{2}$ (or both) are such that the nuclei are polarized (non-zero nuclear orientation), there is an apparent magnetic field associated with the collision, and this field only acts on the nuclear variables $\left({ }^{11}\right)$. The nuclear identity effects are therefore strongly reminiscent of the electron identity effects. Nevertheless, it should be noted that $A_{\text {exch. }}^{S}$ involves interference effects between direct and transfer processes in the same direction of the vector $\mathbf{k}_{\mathrm{f}}$ (see (20)) but $A_{\text {exch. }}^{I}$. for opposite directions (see (37)). This is related to the fact that $P_{\mathrm{n}}$ changes the velocity of both nuclei in the centre of mass reference frame (interference effects associated with scattering processes towards opposite final directions are typical of nuclear identity effects, and analogous results are obtained in the study of metastability exchange collision). This direction change explains why the nucleus identity effects are often less pronounced than the effects of electronic identity [20] : for example, if both $S_{\mathrm{d}}\left(\mathbf{k}_{\mathrm{f}}, \mathbf{k}_{\mathrm{i}}\right)$ and $S_{\mathrm{t}}\left(\mathbf{k}_{\mathrm{f}}, \mathbf{k}_{\mathrm{i}}\right)$ are strongly forward peaked functions (they are supposed to have negligible values if the angle between $\mathbf{k}_{\mathbf{i}}$ and $\mathbf{k}_{\mathrm{f}}$ is not small), $A_{\text {exch. }}^{I}$ is practically zero, but not necessarily $A_{\text {exch. }}^{S}$.

The terms proportional to $E_{I S}$ in (45) involve both the identity effects of electrons and of nuclei together. The terms in $\rho_{1} \times \rho_{2}$ and $\rho_{2} \times \rho_{1}$ have been obtained from the action of the product operator $P_{\mathrm{e}} P_{\mathrm{n}}$. This operator exchanges both constituent particles of the atoms, that is exchanges atoms considered as complete entities (atoms in the etymological meaning). These two terms can therefore be called the atom-exchange terms, and the plus sign arises from the fact that atoms made up of two fermions are bosons $\left({ }^{12}\right)$. The two last terms in (45) are more complex and arise from interference effects between electron and nucleus exchange. As discussed above, their presence exactly cancels the variation of the trace of the density operator due to atomexchange.

Figure 1 shows a schematical representation of the 4 physical processes which influence the density operator evolution. They transform the same initial state with numbered particles into 4 different mathematical kets, all corresponding to the same final physical state for identical electrons and protons. Therefore, interference effects between any pair of these processes are possible. For simplicity, we shall denote them $a, b, c, d$ respectively. The various terms in equation (45) then correspond to the following combination (after an integration over $\theta)$ :

$$
\begin{array}{crr}
a a^{*} \quad b b^{*} & a b^{*}+b^{*} a \\
d d^{*} \quad c c^{*} & c d^{*}+c^{*} d \\
a c^{*}+c^{*} a & b d^{*}+b^{*} d \\
a d^{*}+d^{*} a & b c^{*}+b^{*} c .
\end{array}
$$

We conclude this section by remarking that, if either $\rho_{1}$ or $\rho_{2}$ is proportional to 1 , all particle identity terms in (45) vanish. This is obvious for the terms in $A_{\text {exch. }}^{S}$ and $A_{\text {exch. }}^{I}$ which have the form of commutators. Since :

$$
\begin{aligned}
\left\langle m_{I}, M_{S}\right| \operatorname{Tr}_{2}\left\{P_{\mathrm{n}} \rho_{1}(1) \otimes\right. & \left.\rho_{2}(2) P_{\mathrm{e}}+P_{\mathrm{e}} \rho_{1}(1) \otimes \rho_{2}(2) P_{\mathrm{n}}\right\}\left|m_{I}^{\prime}, M_{S}^{\prime}\right\rangle \\
& =\sum_{m_{I}^{\prime \prime} M_{S}^{\prime \prime}}\left\langle m_{I}^{\prime \prime}, M_{S}\left|\rho_{1}\right| m_{I}^{\prime}, M_{S}^{\prime \prime}\right\rangle\left\langle m_{I}, M_{S}^{\prime \prime}\left|\rho_{2}\right| m_{I}^{\prime \prime}, M_{S}^{\prime}\right\rangle \\
& +\left\langle m_{I}, M_{S}^{\prime \prime}\left|\rho_{1}\right| m_{I}^{\prime \prime}, M_{S}^{\prime}\right\rangle\left\langle m_{I}^{\prime \prime}, M_{S}\left|\rho_{2}\right| m_{I}^{\prime}, M_{S}^{\prime \prime}\right\rangle
\end{aligned}
$$

$\left({ }^{1}{ }^{1}\right)$ If the nuclear spins are greater than $1 / 2$, there is also an apparent electric field gradient acting on the nuclei which depends on the nuclear alignment, etc...

$\left({ }^{12}\right)$ Of course, if the nuclei were particles with integer spin (deuterium for example), a minus sign would be necessary. 


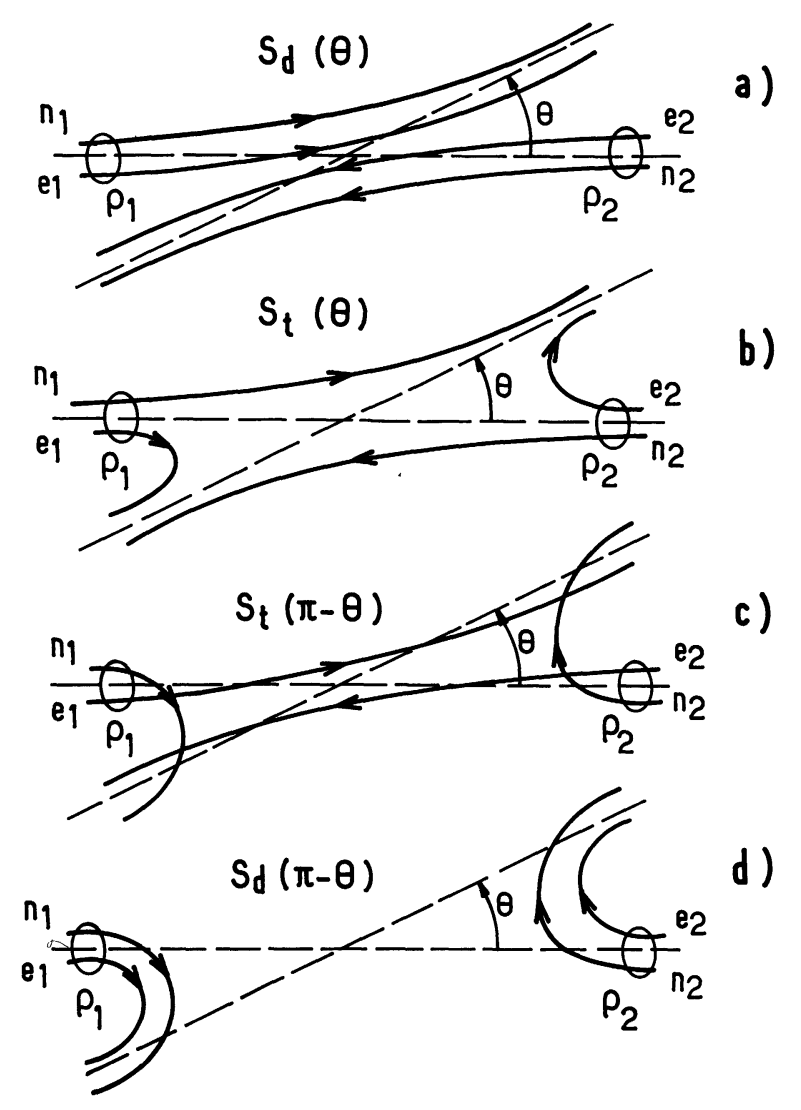

Fig. 1. - Four different scattering processes are schematically represented in diagrams (a), (b), (c) and (d). The particles are labelled : $n_{1}$ and $n_{2}$ are the two nuclei, $e_{1}$ and $e_{2}$ the electrons. $\rho_{1}$ and $\rho_{2}$ are the density operators describing the internal variables of the two atoms before collision. When the particles are distinguishable, these diagrams connect the same initial state to four orthogonal, physically different, final states. For identical nuclei and electrons, the final states become physically indistinguishable, so that interference effects between the diagrams occur. As discussed in the text, these effects introduce additional terms in the equations giving the evolution of the atom internal variables, and produce various apparent magnetic fields acting on the nuclear and electronic spins.

is simply equal to :

$$
2\left\langle m_{I}, M_{S}\left|\rho_{1}\right| m_{I}^{\prime}, M_{S}^{\prime}\right\rangle
$$

if $\rho_{2} \propto \mathbb{1}$, it is easily seen that the terms in $E_{I S}$ also vanish in this case (the same is obviously true if $\rho_{1} \propto \mathbb{1}$ ). Therefore, if :

$$
\begin{aligned}
& \rho_{1} \propto \mathfrak{1}+\Delta \rho_{1} \\
& \rho_{2} \propto 1+\Delta \rho_{2}
\end{aligned}
$$

the exchange terms in $A_{\mathrm{t}}$ are first order in $\Delta \rho_{1}$ and $\Delta \rho_{2}$, but all the other terms are second order (crossed terms in $\Delta \rho_{1}$ and $\left.\Delta \rho_{2}\right)$.

3. Applications to a few special cases. - Let us now apply the equations obtained to a few special cases.

3. 1 COLliSIONS BETWEEN ATOMS WITH THE SAME DENSITY MATRIX. - Equation (45) is general and no assumption has to be made concerning the initial density operators $\rho_{1}$ and $\rho_{2}$. This equation can be used for example to describe the effect of spin exchange collision between a polarized hydrogen beam and a hydrogen gas of atoms having any internal variable state. Nevertheless, in many practical situations (optical pumping experiments with an alkali vapour for example), no correlation exists between the internal state of an atom and its velocity, so that the two initial density operators are simply equal. In (45), if we replace $\rho_{1}$ and $\rho_{2}$ by $\rho_{\mathrm{i}}$, we obtain :

$$
\begin{aligned}
\rho_{\mathrm{f}}(\mathrm{e}, \mathrm{n})=\left(1-A_{\mathrm{t}}\right) \rho_{\mathrm{i}}+A_{\mathrm{t}} \operatorname{Tr}_{S}\left\{\rho_{\mathrm{i}}\right\} \otimes & \operatorname{Tr}_{I}\left\{\rho_{\mathrm{i}}\right\} \\
& -i A_{\mathrm{exch} .}^{S}\left[\rho_{\mathrm{i}}, \mathbb{V}_{I} \otimes \operatorname{Tr}_{I}\left\{\rho_{\mathrm{i}}\right\}\right]-i A_{\text {exch. }}^{I}\left[\rho_{\mathrm{i}}, \operatorname{Tr}_{S}\left\{\rho_{\mathrm{i}}\right\} \otimes \mathbb{V}_{S}\right] \\
& -E_{I S}\left(\rho_{\mathrm{i}}^{2}-\operatorname{Tr}_{2}\left\{P_{\mathrm{n}} \rho_{\mathrm{i}}(1) \otimes \rho_{\mathrm{i}}(2) P_{\mathrm{e}}\right\}\right) .
\end{aligned}
$$

To write this equation, we have used the equality [only valid if $\rho_{1}=\rho_{2}=\rho_{\mathrm{i}}$ ] :

$$
\operatorname{Tr}_{2}\left\{P_{\mathrm{n}} \rho_{\mathrm{i}}(1) \otimes \rho_{\mathrm{i}}(2) P_{\mathrm{e}}\right\}=\operatorname{Tr}_{2}\left\{P_{\mathrm{e}} \rho_{\mathrm{i}}(1) \otimes \rho_{\mathrm{i}}(2) P_{\mathrm{n}}\right\}
$$

which can easily be shown from (48). 
We have already checked in the general case $\left(\rho_{1} \neq \rho_{2}\right)$ that the trace of $\rho_{\mathrm{f}}$ is equal to the trace of $\rho_{\mathrm{i}}$. Other quantities which are conserved are the angular momenta $\langle\mathbf{I}\rangle$ and $\langle\mathbf{S}\rangle$ (and therefore $\langle\mathbf{F}\rangle$ ). It is practically obvious on (49) that the sum of the terms in $\left(1-A_{t}\right)$ and $A_{t}$ has this property. In addition, this is also true for the exchange terms in (49), since :

$$
\begin{aligned}
\operatorname{Tr}\left\{\mathbf{S} \rho_{\mathrm{i}} \mathbb{1}_{I} \otimes \operatorname{Tr}_{I}\left\{\rho_{\mathrm{i}}\right\}\right\}=\operatorname{Tr}_{S}\left\{\mathbf{S} \operatorname{Tr}_{I}\left\{\rho_{\mathrm{i}} \mathbb{1}_{I} \otimes \operatorname{Tr}_{I}\left\{\rho_{\mathrm{i}}\right\}\right\}\right\}=\operatorname{Tr}_{S}\left\{\mathbf{S} \operatorname{Tr}_{I}\left\{\rho_{\mathrm{i}}\right\} \times \operatorname{Tr}_{I}\left\{\rho_{\mathrm{i}}\right\}\right\} \\
=\operatorname{Tr}\left\{\mathbf{S} \mathbb{1}_{I} \otimes \operatorname{Tr}_{I}\left\{\rho_{\mathrm{i}}\right\} \rho_{\mathrm{i}}\right\}
\end{aligned}
$$

which shows that $\langle\mathbf{S}\rangle$ is not affected by the commutator in $A_{\text {exch. }}^{S}$. On the other hand, I commutes with an electronic operator and $\langle\mathbf{I}\rangle$ is not affected either. The same reasoning being clearly possible when $\mathbf{I}$ and $\mathbf{S}$ are interchanged, so that neither of the terms in $A_{\text {exch. }}^{S}$ or $A_{\text {exch. }}^{I}$ affect $\langle\mathbf{I}\rangle$ and $\langle\mathbf{S}\rangle$. Finally, the term in $E_{I S}$ also has this property :

$$
\begin{array}{r}
\operatorname{Tr}_{1}\left\{\mathrm{~S}(1) \operatorname{Tr}_{2}\left\{P_{\mathrm{n}} \rho_{\mathrm{i}}(1) \otimes \rho_{\mathrm{i}}(2) P_{\mathrm{e}}\right\}\right\}=\sum_{m_{I} M_{S}} \hbar M_{S} \sum_{m_{I}^{\prime} M_{S}^{\prime}}\left\langle m_{I}^{\prime} M_{S}\left|\rho_{\mathrm{i}}\right| m_{I} M_{S}^{\prime}\right\rangle\left\langle m_{I} M_{S}^{\prime}\left|\rho_{\mathrm{i}}\right| m_{I}^{\prime} M_{S}\right\rangle \\
=\operatorname{Tr}_{1}\left\{\mathbf{S}(1) \rho_{\mathrm{i}}^{2}(1)\right\} .
\end{array}
$$

Physically, since we have assumed that the collision does not act on the spin variables, it is satisfying that $\langle\mathbf{I}\rangle$ and $\langle\mathbf{S}\rangle$ are conserved.

Equation (49) can be compared to equation (B .2) in Appendix B of reference [9], with the correspondence :

$$
\begin{aligned}
& \rho(H, \bar{H})=\rho_{\mathrm{i}}(1) \otimes \rho_{\mathrm{i}}(2) \\
& \sigma(H) . \sigma(\bar{H})=2 P_{\mathrm{e}}-1 \\
& \sigma_{S F} \propto A_{\mathrm{t}} \\
& \kappa \sigma_{S F} \propto A_{\text {exch. }}^{S} \\
& \sigma_{S F}^{\prime} \propto E_{I S} \\
& \kappa^{\prime} \sigma_{S F}^{\prime} \propto A_{\text {exch. }}^{I}
\end{aligned}
$$

(for a more precise comparison of these four numerical coefficients, see the Appendix of the present article). One has to take the trace of equation (B.2) over the $\bar{H}$ variables. This calculation gives a first contribution equal to :

$$
\begin{gathered}
\operatorname{Tr}_{2}\left\{-3 \rho_{\mathrm{i}}(1) \otimes \rho_{\mathrm{i}}(2)+(1+2 i \kappa)\left[2 P_{\mathrm{e}}-1\right]\left[\rho_{\mathrm{i}}(1) \otimes \rho_{\mathrm{i}}(2)\right]+(1-2 i \kappa)\left[\rho_{\mathrm{i}}(1) \otimes \rho_{\mathrm{i}}(2)\right]\left[2 P_{\mathrm{e}}-1\right]\right. \\
\left.+\left[2 P_{\mathrm{e}}-1\right]\left[\rho_{\mathrm{i}}(1) \otimes \rho_{\mathrm{i}}(2)\right]\left[2 P_{\mathrm{e}}-1\right]\right\} \\
=-3 \rho_{\mathrm{i}}-(1+2 i \kappa) \rho_{\mathrm{i}}-(1-2 i \kappa) \rho_{\mathrm{i}}+\rho_{\mathrm{i}} \\
+4 i \kappa \operatorname{Tr}_{2}\left\{\left[P_{\mathrm{e}}, \rho_{\mathrm{i}}(1) \otimes \rho_{\mathrm{i}}(2)\right]\right\}+4 \operatorname{Tr}_{2}\left\{P_{\mathrm{e}} \rho_{\mathrm{i}}(1) \otimes \rho_{\mathrm{i}}(2) P_{\mathrm{e}}\right\} \\
=-4 \rho_{\mathrm{i}}+4 \operatorname{Tr}_{I}\left\{\rho_{\mathrm{i}}\right\} \otimes \operatorname{Tr}_{S}\left\{\rho_{\mathrm{i}}\right\}+2 i \kappa \operatorname{Tr}_{2}\left\{\left[\sigma(1) . \sigma(2), \rho_{\mathrm{i}}(1) \otimes \rho_{\mathrm{i}}(2)\right]\right\}
\end{gathered}
$$

Since :

$$
\operatorname{Tr}_{2}\left\{\left[\sigma(1) \cdot \sigma(2), \rho_{\mathrm{i}}(1) \otimes \rho_{\mathrm{i}}(2)\right]\right\}=\langle\sigma\rangle_{2} \cdot\left[\sigma(1), \rho_{\mathrm{i}}(1)\right],
$$

we obtain results identical to three first terms of the right hand side of (49) (see also (31b)). The terms written in (51) therefore contain the transfer effect and the electron indistinguishability effect. A similar agreement can be found for the nucleus indistinguishability terms [terms proportional to $\sigma_{S F}^{\prime}$ ] :

$$
\begin{aligned}
\operatorname{Tr}_{2}\left\{-3 \rho_{\mathrm{i}}(1) \otimes \rho_{\mathrm{i}}(2) P_{\mathrm{e}} P_{\mathrm{n}}+\left(1+2 i \kappa^{\prime}\right)\left[2 P_{\mathrm{e}}-1\right] P_{\mathrm{e}} P_{\mathrm{n}}\left[\rho_{\mathrm{i}}(1) \otimes \rho_{\mathrm{i}}(2)\right]\right. \\
\left.\quad+\left(1-2 i \kappa^{\prime}\right)\left[\rho_{\mathrm{i}}(1) \otimes \rho_{\mathrm{i}}(2)\right] P_{\mathrm{e}} P_{\mathrm{n}}\left[2 P_{\mathrm{e}}-1\right]+\left[2 P_{\mathrm{e}}-1\right] P_{\mathrm{e}} P_{\mathrm{n}}\left[\rho_{\mathrm{i}}(1) \otimes \rho_{\mathrm{i}}(2)\right]\left[2 P_{\mathrm{e}}-1\right]\right\} \\
=-3 \rho_{\mathrm{i}}^{2}-\left(1+2 i \kappa^{\prime}\right) \rho_{\mathrm{i}}^{2}-\left(1-2 i \kappa^{\prime}\right) \rho_{\mathrm{i}}^{2}+\rho_{\mathrm{i}}^{2} \\
\quad+4 i \kappa^{\prime} \operatorname{Tr}_{2}\left\{\left[P_{\mathrm{n}}, \rho_{\mathrm{i}}(1) \otimes \rho_{\mathrm{i}}(2)\right]\right\}+4 \operatorname{Tr}_{2}\left\{P_{\mathrm{n}} \rho_{\mathrm{i}}(1) \otimes \rho_{\mathrm{i}}(2) P_{\mathrm{e}}\right\}
\end{aligned}
$$

We have made use of the fact that [see (39)] :

$$
\begin{aligned}
& \operatorname{Tr}_{2}\left\{\rho_{1}(1) \otimes \rho_{2}(2) P_{\mathrm{e}} P_{\mathrm{n}}\right\}=\rho_{1} \times \rho_{2} \\
& \operatorname{Tr}_{2}\left\{P_{\mathrm{e}} P_{\mathrm{n}} \rho_{1}(1) \otimes \rho_{2}(2)\right\}=\rho_{2} \times \rho_{1} .
\end{aligned}
$$


The term in $-4 \rho_{\mathrm{i}}^{2}$ corresponds to the first term proportional to $E_{I S}$ in (49), the term in $\operatorname{Tr}_{2}\left\{P_{\mathrm{n}} \rho_{\mathrm{i}}(1) \otimes \rho_{\mathrm{i}}(2) P_{\mathrm{e}}\right\}$ to the second term proportional to $E_{I S}$. As for the term in $\kappa^{\prime}$, a reasoning similar to the one which gave (52) shows that it is the nuclear identity term [proportional to $A_{\text {exch. }}^{I}$ in (49)].

Remark. - If $\rho_{1} \neq \rho_{2}$, the development of equation (B.2) of reference [9] gives a result in which neither the terms in $\sigma_{S F}$ nor the terms in $\sigma_{S F}^{\prime}$ are invariant when $\rho_{1}$ and $\rho_{2}$ are interchanged. For example, (53) becomes :

$$
\begin{aligned}
-3 \rho_{1} \times \rho_{2}-\left(1+2 i \kappa^{\prime}\right) \rho_{2} \times \rho_{1}-(1 & \left.-2 i \kappa^{\prime}\right) \rho_{1} \times \rho_{2}+\rho_{2} \times \rho_{1} \\
& +4 i \kappa^{\prime} \operatorname{Tr}_{2}\left\{\left[P_{\mathrm{n}}, \rho_{1}(1) \otimes \rho_{2}(2)\right]\right\}+4 \operatorname{Tr}_{2}\left\{P_{\mathrm{n}} \rho_{1}(1) \otimes \rho_{2}(2) P_{\mathrm{e}}\right\} \\
& +2 \operatorname{Tr}_{2}\left\{\rho_{1}(1) \otimes \rho_{2}(2) P_{\mathrm{n}}-P_{\mathrm{e}} P_{\mathrm{n}} \rho_{1}(1) \otimes \rho_{2}(2) P_{\mathrm{e}}\right\}
\end{aligned}
$$

which does not coincide with our result (45). We therefore think that the validity of equation (B .2) of reference [9] is restricted to the case when the internal state of the colliding atoms is the same. Otherwise, equation (45) should be used.

3.2 SPIN EXCHANGE SHIFT OF THE 0-0 HYPERFINE COHERENCE. - As an illustration of the simplification obtained when using equation (48) instead of relationships involving $16 \times 16$ matrices, we shall calculate the effect of spin exchange collisions between hydrogen atoms on their ground state 0-0 hyperfine coherence. We assume here that :

$$
\rho_{\mathrm{i}}=\left(\begin{array}{ccc:c}
c & F=1 & F=0 \\
0 & 0 & 0 & 0 \\
0 & 0 & c & 0 \\
\hdashline 0 & x-i y & 0 & d y
\end{array}\right)
$$

[the order of the states being $|F=1, M=1\rangle,|F=1, M=0\rangle,|F=1, M=-1\rangle,|F=0, M=0\rangle$; we use the same notation as reference [11], with $j=x-i y]$ with :

$$
a+b+c+d=1 .
$$

In the decoupled basis $\left|m_{I} M_{S}\right\rangle$, this matrix becomes :

$$
\rho_{\mathrm{i}}=\frac{1}{2}\left(\begin{array}{cccc}
2 a & |+-\rangle & |-+\rangle & |--\rangle \\
0 & b+d+2 x & b-d-2 i y & 0 \\
0 & b-d+2 i y & b+d-2 x & 0 \\
0 & 0 & 0 & 2 c
\end{array}\right) .
$$

It is then easy to see that :

$$
\left\{\begin{array}{l}
\left\langle I_{z}\right\rangle=\frac{1}{2}[a-c+2 x] \\
\left\langle S_{z}\right\rangle=\frac{1}{2}[a-c-2 x] \\
\left\langle F_{z}\right\rangle=[a-c]
\end{array}\right.
$$

(I, $\mathbf{S}$ and $\mathbf{F}$ are respectively the nuclear spin, electron spin, and total spin divided by $\hbar$ ).

Taking the two partial traces of $(55 c)$ leads to :

and

$$
\rho_{I}=\operatorname{Tr}_{S}\left\{\rho_{\mathrm{i}}\right\}=\left(\begin{array}{cc}
\frac{1}{2}+\left\langle I_{z}\right\rangle & 0 \\
0 & \frac{1}{2}-\left\langle I_{z}\right\rangle
\end{array}\right)
$$

$$
\rho_{S}=\operatorname{Tr}_{I}\left\{\rho_{\mathrm{i}}\right\}=\left(\begin{array}{cc}
\frac{1}{2}+\left\langle S_{z}\right\rangle & 0 \\
0 & \frac{1}{2}-\left\langle S_{z}\right\rangle
\end{array}\right)
$$


(no coherence is left in $\rho_{I}$ and $\rho_{S}$ in this particular case). A straightforward calculation then gives $\rho_{I} \otimes \rho_{S}$ and, coming back to the $\left|F, M_{F}\right\rangle$ basis, we obtain :

$\rho_{I} \otimes \rho_{S}=\frac{1}{4}\left(\begin{array}{ccc:c}{\left[1+\left\langle F_{z}\right\rangle\right]^{2}-4 x^{2}} & 0 & 0 & 0 \\ 0 & {\left[1-\left\langle F_{z}\right\rangle^{2}\right]+4 x^{2}} & 0 & x \\ 0 & 0 & {\left[1-\left\langle F_{z}\right\rangle\right]^{2}-4 x^{2}} & 0 \\ \hdashline 0 & x & 0 & {\left[1-\left\langle F_{z}\right\rangle^{2}\right]+4 x^{2}}\end{array}\right)$

This matrix gives the density operator just after collision when all particle identity effects are ignored (i.e. only the terms proportional to $\left.\sigma_{S F} \propto A_{\mathrm{t}}\right)$. It gives a generalization of the results of Appendix II of reference [8] to the case when $\left\langle F_{z}\right\rangle \neq 0$. Similar calculations give :

$$
\begin{gathered}
{\left[\rho_{\mathrm{i}}, 0_{I} \otimes \rho_{S}\right]=\left\langle S_{z}\right\rangle\left(\begin{array}{ccc:c}
0 & 0 & 0 & 0 \\
0 & -2 i y & 0 & d-b \\
0 & 0 & 0 & 0 \\
\hdashline 0 & b-d & 0 & 2 i y
\end{array}\right)} \\
{\left[\rho_{\mathrm{i}}, \rho_{I} \otimes 1 \mathbb{1}_{s}\right]=\left\langle I_{z}\right\rangle\left(\begin{array}{ccc:c}
0 & 0 & 0 & 0 \\
0 & 2 i y & 0 & b-d \\
0 & 0 & 0 & 0 \\
\hdashline 0 & d-b & 0 & -2 i y
\end{array}\right)} \\
\rho_{\mathrm{i}} \times \rho_{\mathrm{i}}=\left(\begin{array}{cccc}
a^{2} & 0 & 0 & 0 \\
0 & b^{2}+x^{2}+y^{2} & 0 & (b+d)(x+i y) \\
0 & 0 & c^{2} & \\
\hdashline 0 & (b+d)(x-i y) & 0 & d^{2}+x^{2}+y^{2}
\end{array}\right) .
\end{gathered}
$$

and finally :

$$
\begin{aligned}
& \operatorname{Tr}_{2}\left\{P_{\mathrm{n}} \rho_{\mathrm{i}}(1) \otimes \rho_{\mathrm{i}}(2) P_{\mathrm{e}}\right\}=\operatorname{Tr}_{2}\left\{P_{\mathrm{e}} \rho_{\mathrm{i}}(1) \otimes \rho_{\mathrm{i}}(2) P_{\mathrm{n}}\right\} \\
& = \\
& =\left(\begin{array}{cccc}
a^{2}+\left(\frac{b-d}{2}\right)^{2}+y^{2} & 0 & 0 & 0 \\
0 & \left(\frac{b+d}{2}\right)^{2}+\frac{(a+c)(b-d)}{2}+x^{2} & 0 & x(b+d)+i y(a+c) \\
0 & 0 & c^{2}+\left(\frac{b-d}{2}\right)^{2}+y^{2} & 0 \\
0 & x(b+d)-i y(a+c) & 0 & \left(\frac{b+d}{2}\right)^{2}+x^{2}-\frac{(a+c)(b-d)}{2}
\end{array}\right) .
\end{aligned}
$$

As expected, the electron and nucleus identity terms are proportional to $\left\langle S_{z}\right\rangle$ and $\left\langle I_{z}\right\rangle$ respectively (apparent magnetic fields proportional to the orientation). The crossed exchange terms have a more complex structure as shown by $(58 d)$ and $(58 e)$. From these equations, it is possible to obtain the 0-0 hyperfine coherence $(x+i y)_{\mathrm{f}}$ just after collision

$$
(x+i y)_{\mathrm{f}}=\left(1-A_{\mathrm{t}}\right)(x+i y)+A_{\mathrm{t}} x+i A_{\text {exch. }}^{S}\left\langle S_{z}\right\rangle(b-d)-i A_{\text {exch. }}^{I}\left\langle I_{z}\right\rangle(b-d)+i E_{I S} y(a+c-b-d) .
$$

The first two terms in the right hand side of this equation show that, when a spin transfer occurs (term in $A_{t}$ ), the hyperfine coherence $x+i y$ is simply replaced by its real part $x$, the imaginary part $i y$ being completely destroyed. This can be understood physically from the equations :

$$
\begin{aligned}
& x=\left\langle I_{z}\right\rangle-\left\langle S_{z}\right\rangle \\
& y \propto \operatorname{Im}\left\langle I_{-} S_{+}\right\rangle=\left\langle I_{x} S_{y}-I_{y} S_{x}\right\rangle=\left\langle(\mathbf{I} \times \mathbf{S})_{z}\right\rangle
\end{aligned}
$$


which show that $y$, but not $x$, depends on the correlations between $\mathbf{I}$ and $\mathbf{S}$ variables. We have seen earlier that $\langle\mathbf{I}\rangle$ and $\langle\mathbf{S}\rangle$ are not affected by the collision which explains the complete conservation of $x$. On the other hand, after a process where $\mathbf{I}$ and $\mathbf{S}$ are decorrelated, $\left\langle I_{x} S_{y}-I_{y} S_{x}\right\rangle$ is replaced by $\left\langle I_{x}\right\rangle\left\langle S_{y}\right\rangle-\left\langle I_{y}\right\rangle\left\langle S_{x}\right\rangle$. Since the density operator $(55 a)$ is invariant under any rotation about $\mathrm{O} z$, this quantity clearly vanishes.

One can also see on (59) that all particle identity exchange terms (in $A_{\text {exch. }}^{I}, A_{\text {exch. }}^{S}$ and $E_{I S}$ ) vanish if there is no initial population difference.

The time dependence of the populations $a, b, c$ and $d$, and of the hyperfine coherence $x+i y$, are very different : the former changes at a much slower rate than the latter (the hyperfine frequency is supposed to be much larger than the atom exchange rate, the inverse relaxation or pumping time, etc...). Therefore, we must distinguish in (59) terms which are secular (or resonant) and can have a significant effect, from non-secular terms. Let us for example first discuss the term in $A_{\text {exch. }}^{S}$, which is proportional to $\left\langle S_{z}\right\rangle$. The (quasi) static part $\left\langle S_{z}\right\rangle_{\text {st. }}$ of $\left\langle S_{z}\right\rangle$ [the part which depends on the populations $a$ and $c$; see (56b)] gives a non-resonant coupling between the hyperfine coherence and populations and its effect can be neglected (it is well known that a static magnetic field does not affect the eigenfrequency of the 0-0 hyperfine coherence to first order). But, as noted in paragraph VI. 6 of reference [2], $\left\langle S_{z}\right\rangle$ also comprises a time dependent part $\left\langle S_{z}\right\rangle_{\text {osc. }}(t)$ which oscillates at the 0-0 hyperfine frequency, that is exactly the frequency needed to resonantly couple $x+i y$ and populations. The physical interpretation of the term :

$$
i A_{\text {exch. }}^{S}\left\langle S_{z}\right\rangle_{\text {osc. }}(t)[b-d]
$$

is then clear : the apparent oscillating magnetic field proportional to $\left\langle S_{z}\right\rangle_{\text {osc. }}(t)$ (and parallel to $\mathrm{O} z$ ) produces a magnetic resonance effect between the two levels of populations $b$ and $d(0-0$ hyperfine resonance). This explains why the coherence is only coupled to the population difference of the two levels involved, as is well known in magnetic resonance. The term in $A_{\text {exch. }}^{I}$ can be interpreted in the same way, the only difference being that the apparent field is now proportional to $\left\langle I_{z}\right\rangle_{\text {osc. }}(t)$ and only acts on $\mathbf{I}$ (not on $\mathbf{S}$, in contrast to what a real field would mostly do). The last term in (59), proportional to $E_{I S}$, is the only one which depends on the imaginary part $y$ of the hyperfine coherence.

Equalities $(56 a)$ and $(56 b)$ clearly show the low frequency (terms in $a-c)$ and high frequency (terms in $x$ ) parts of $\left\langle I_{z}\right\rangle$ and $\left\langle S_{z}\right\rangle$ and since :

$$
\begin{aligned}
& x=\frac{1}{2}(x+i y)+\frac{1}{2}(x-i y) \\
& y=\frac{1}{2 i}(x+i y)-\frac{1}{2 i}(x-i y),
\end{aligned}
$$

equation (59) can be re-written :

$$
\begin{array}{r}
(x+i y)_{\text {coll. }}-(x+i y)=-\frac{A_{\mathrm{t}}}{2}(x+i y)-i A_{\text {exch. }}^{S}(b-d) \frac{x+i y}{2}-i A_{\text {exch. }}^{I}(b-d) \frac{x+i y}{2} \\
+E_{I S}(a+c-b-d) \frac{x+i y}{2}+\text { non-secular terms }
\end{array}
$$

Therefore, the frequency shift of the hyperfine coherence due to spin exchange collision is [we use the same notation as in equation (29)] :

$$
\Delta v_{\text {exch. }}=\frac{1}{2 \pi T_{\text {coll. }}}\left\{\frac{b-d}{2}\left(\kappa^{S}+\kappa^{I}\right)\right\}
$$

where :

$$
\kappa_{S}=\frac{A_{\text {exch. }}^{S}}{A_{\mathrm{t}}} \quad \kappa_{\mathrm{I}}=\frac{A_{\mathrm{exch}}^{I}}{A_{\mathrm{t}}}
$$

(see Appendix for a precise calculation of these coefficients). Equations (60a) and (60b) exactly coincide with the results available from table $\mathrm{X}$ of reference [9]. 
3.3 SPIN EXCHANGE SHIFTS OF ZEEMAN COHERENCES. - Another interesting situation occurs when there are non-zero Zeeman coherences inside the hyperfine $F=1$ sublevel :

$$
\rho_{\mathrm{i}}=\left(\begin{array}{ccc:c}
a & e^{*} & f^{*} & 0 \\
e & b & g^{*} & 0 \\
f & g & c & 0 \\
\hdashline 0 & 0 & 0 & d
\end{array}\right)
$$

[again we use the notation of reference [11]]. In the decoupled basis $\left|m_{I} M_{S}\right\rangle$, this matrix becomes :

$$
\begin{gathered}
|++\rangle \\
\rho_{\mathrm{i}}=\left(\begin{array}{cccc}
a & |+-\rangle & |-+\rangle & |--\rangle \\
e / \sqrt{2} & (b+d) / 2 & (b-d) / 2 & g^{*} / \sqrt{2} \\
e / \sqrt{2} & (b-d) / 2 & (b+d) / 2 & g^{*} / \sqrt{2} \\
f & g / \sqrt{2} & g / \sqrt{2} & c
\end{array}\right) .
\end{gathered}
$$

One then finds :

$$
\rho_{I}=\operatorname{Tr}_{S}\left\{\rho_{\mathrm{i}}\right\}=\left(\begin{array}{cc}
a+(b+d) / 2 & \left(e^{*}+g^{*}\right) / \sqrt{2} \\
(e+g) / \sqrt{2} & c+(b+d) / 2
\end{array}\right)
$$

and :

$$
\rho_{S}=\operatorname{Tr}_{I}\left\{\rho_{\mathrm{i}}\right\}=\rho_{I}
$$

$\left[\rho_{I}=\rho_{S}\right.$ because $\mathbf{I}$ and $\mathbf{S}$ are proportional operators inside the $F=1$ sublevel, according to the Wigner-Eckart theorem]. Equations (56) must now be replaced by :

$$
\left.\begin{array}{l}
\left\langle I_{z}\right\rangle=\left\langle S_{z}\right\rangle=\frac{1}{2}[a-c] \\
\left\langle I_{+}\right\rangle=\left\langle S_{+}\right\rangle=[e+g] / \sqrt{2}
\end{array}\right\}
$$

where $I_{ \pm}$and $S_{ \pm}$are defined by :

$$
\left\{\begin{array}{l}
I_{ \pm}=I_{x} \pm i I_{y} \\
S_{ \pm}=S_{x} \pm i S_{y}
\end{array}\right.
$$

One then obtains (in the $\left|F M_{F}\right\rangle$ basis) :

$\rho_{I} \otimes \rho_{S}=$

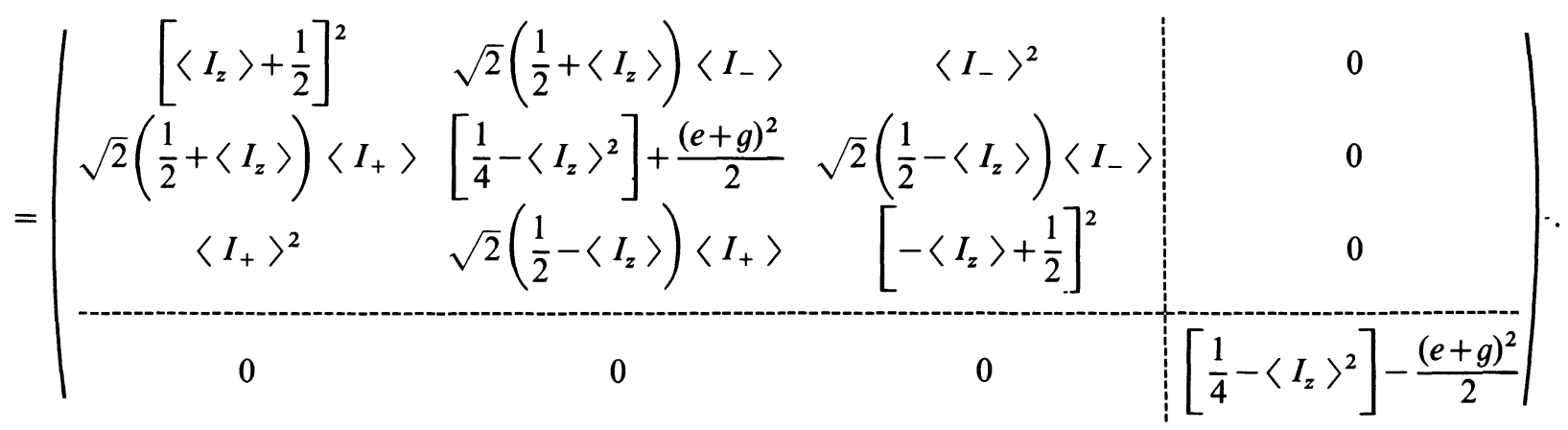

This matrix gives the term in $A_{\mathrm{t}}$ in the right hand side of (49). Similar calculations can be performed for the terms in $A_{\text {exch. }}^{S}, A_{\text {exch. }}^{I}$ and $E_{I S}$ but will not be reproduced here. Let us only calculate the mean value after collision of $\left\langle F_{z}\right\rangle$ and $\left\langle 3 F_{z}^{2}-F(F+1)\right\rangle$ (longitudinal orientation and alignment) and of $\left\langle F_{ \pm}\right\rangle,\left\langle F_{z} F_{ \pm}+F_{ \pm} F_{z}\right\rangle$ and $\left\langle F_{ \pm}^{2}\right\rangle$ (transverse orientation and alignment). 
We can check on (64) that :

$$
\begin{aligned}
& \left\langle F_{z}\right\rangle_{\mathrm{f}}=\left\langle F_{z}\right\rangle_{\mathrm{i}} \\
& \left\langle F_{ \pm}\right\rangle_{\mathrm{f}}=\left\langle F_{ \pm}\right\rangle_{\mathrm{i}}
\end{aligned}
$$

(the total angular momentum is conserved in the collision). One also obtains :

$$
\begin{aligned}
& \left\langle 3 F_{z}^{2}-F(F+1)\right\rangle_{\mathrm{f}}=\left(1-A_{\mathrm{t}}\right)\left\langle 3 F_{z}^{2}-F(F+1)\right\rangle_{\mathrm{i}}+A_{\mathrm{t}}\left[\left\langle F_{z}\right\rangle_{\mathrm{i}}^{2}-\left\langle F_{+}\right\rangle_{\mathrm{i}}\left\langle F_{-}\right\rangle_{\mathrm{i}} / 2\right] \\
& \quad-3 i\left(A_{\text {exch. }}^{S}+A_{\text {exch. }}^{I}\right)\left[e^{*} g-e g^{*}\right]-E_{I S}\left[4|f|^{2}-|e|^{2}-|g|^{2}+(d-b)(2 b-a-c)+e^{*} g+e g^{*}\right]
\end{aligned}
$$

$$
\begin{aligned}
\left\langle F_{z} F_{+}\right. & \left.+F_{+} F_{z}\right\rangle_{\mathrm{f}}=\left(1-A_{\mathrm{t}}\right)\left\langle F_{z} F_{+}+F_{+} F_{z}\right\rangle_{\mathrm{i}}+A_{\mathrm{t}}\left\langle F_{z}\right\rangle_{\mathrm{i}}\left\langle F_{+}\right\rangle_{\mathrm{i}} \\
& +\frac{i}{2}\left(A_{\text {exch. }}^{S}+A_{\text {exch. }}^{I}\right)\left[(a+c-2 b)(e+g)+2 f\left(e^{*}+g^{*}\right)-(e-g)(a-c)\right]-E_{I S}\left[2 f\left(g^{*}-e^{*}\right)+(g-e)(d-b)\right]
\end{aligned}
$$

$\left\langle F_{+}^{2}\right\rangle_{\mathrm{f}}=\left(1-A_{\mathrm{t}}\right)\left\langle F_{+}^{2}\right\rangle_{\mathrm{i}}+A_{\mathrm{t}}\left\langle F_{+}\right\rangle_{\mathrm{i}}^{2} / 2$

$$
+i\left(A_{\text {exch. }}^{S}+A_{\text {exch. }}^{I}\right)\left[\frac{\left(e^{2}-g^{2}\right)}{2}+f(c-a)\right]-E_{I S}\left[f(2 a+2 c-1)-\frac{1}{2}(f-g)^{2}\right] \text {. }
$$

These results show how the various Zeeman coherences are resonantly coupled by the exchange processes (the rotation invariance ensures that each observable inside the $F=1$ sublevel is only coupled to other observables in this sublevel having the same Zeeman evolution frequency). The terms in $A_{\mathrm{t}}$ can be understood in terms of complete decorrelation between $\mathbf{I}$ and $\mathbf{S}$ variables. For example, since :

$$
F_{+}^{2}=\left(I_{+}+S_{+}\right)^{2}=2 I_{+} S_{+}
$$

$\left(I_{+}^{2}\right.$ and $S_{+}^{2}$ are identically zero for spin $1 / 2$ particles), $\left\langle F_{+}^{2}\right\rangle$ is replaced in a transfer collision by :

$$
2\left\langle I_{+}\right\rangle_{\mathrm{i}}\left\langle S_{+}\right\rangle_{\mathrm{i}}=\frac{1}{2}\left\langle F_{+}\right\rangle_{\mathrm{i}}^{2}
$$

(I and $\mathbf{S}$ are proportional to $\mathbf{F}$ inside the $F=1$ hyperfine sublevel according to the Wigner-Eckart theorem). In the same way :

$$
F_{z} F_{+}+F_{+} F_{z}=2\left(I_{z} S_{+}+S_{z} I_{+}\right)
$$

$\left(I_{z} I_{+}+I_{+} I_{z}\right.$ and $S_{z} S_{+}+S_{+} S_{z}$ are identically zero) and $\left\langle F_{z} F_{+}+F_{+} F_{z}\right\rangle$ is replaced by :

$$
2\left\langle I_{z}\right\rangle_{\mathrm{i}}\left\langle S_{+}\right\rangle_{\mathrm{i}}+2\left\langle S_{z}\right\rangle_{\mathrm{i}}\left\langle I_{+}\right\rangle_{\mathrm{i}}=\left\langle F_{z}\right\rangle_{\mathrm{i}}\left\langle F_{+}\right\rangle_{\mathrm{i}}
$$

We see on equations (65) that the term in $A_{\text {exch. }}^{S}$ has the same form as the term in $A_{\text {exch. }}^{I}$ This is because I and $\mathbf{S}$ can be replaced by $\mathbf{F} / 2$ as long as we are only interested in observables inside the $F=1$ sublevels. Both electron and nucleus identity terms therefore correspond to a rotation of these observables around $\langle\mathbf{F}\rangle$ (in this way, we find again that $\langle\mathbf{F}\rangle$ is conserved). Experimentally, the fact that, inside the $F=1$ sublevel, only alignment variables are modified by particle identity effects can be used to isolate these effects : one can for example measure the difference between magnetic resonance frequencies for orientations and for alignment.

3.4 COMPLETE HYPERFINE DECOUPLING. - As a last example, let us study the effect of spin exchange collisions in experiments where a static magnetic field produces a complete decoupling of $\mathbf{I}$ and $\mathbf{S}$ (cf. Fig. 2). This kind of situation can occur for instance in experiments where one wants to produce spin polarized $H$ atoms in a high field [21, 22, 23]. It has been considered already in the literature [24], but some of the results we shall obtain are different. 


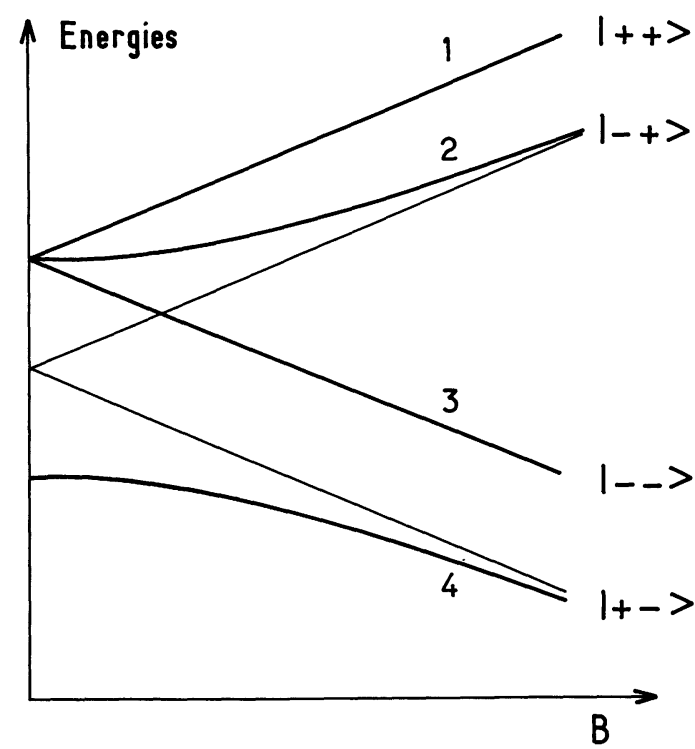

Fig. 2. - Energies of the $\left|m_{I} M_{S}\right\rangle$ substates of a ground state hydrogen atom in a magnetic field $B$. The levels are numbered in order of decreasing energies (although, at extremely high fields, levels 1 and 2 cross and their order is reversed).

We now assume that the initial density matrix in the decoupled basis $\left|m_{I} M_{S}\right\rangle$ has the form $\left({ }^{13}\right)$ (we use the $\xi$ notation of reference [24]) :

$$
\rho_{\mathrm{i}}=\left(\begin{array}{cccc}
|++\rangle & |-+\rangle & |--\rangle & |+-\rangle \\
\frac{1}{4}+\xi_{1} & \theta_{12} & \theta_{13} & \theta_{14} \\
\theta_{12}^{*} & \frac{1}{4}+\xi_{2} & \theta_{23} & \theta_{24} \\
\theta_{13}^{*} & \theta_{23}^{*} & \frac{1}{4}+\xi_{3} & \theta_{34} \\
\theta_{14}^{*} & \theta_{24}^{*} & \theta_{34}^{*} & \frac{1}{4}+\xi_{4}
\end{array}\right)
$$

with :

$$
\xi_{1}+\xi_{2}+\xi_{3}+\xi_{4}=0
$$

The partial traces $\rho_{I}$ and $\rho_{S}$ are equal to (the order of the base states is $|+\rangle,|-\rangle$ );

$$
\begin{aligned}
\rho_{I} & =\left(\begin{array}{cc}
\frac{1}{2}+\xi_{1}+\xi_{4} & \theta_{12}+\theta_{34}^{*} \\
\theta_{12}^{*}+\theta_{34} & \frac{1}{2}+\xi_{2}+\xi_{3}
\end{array}\right) \\
\rho_{S} & =\left(\begin{array}{cc}
\frac{1}{2}+\xi_{1}+\xi_{2} & \theta_{14}+\theta_{23} \\
\theta_{14}^{*}+\theta_{23}^{*} & \frac{1}{2}+\xi_{3}+\xi_{4}
\end{array}\right) .
\end{aligned}
$$

We then obtain, still in the same base as in (66) :

$$
\rho_{I} \otimes \rho_{S}=\rho_{\mathrm{i}}+\frac{1}{2}\left(\begin{array}{cccc}
\left(\xi_{2}+\xi_{4}\right) & \left(\theta_{34}^{*}-\theta_{12}\right) & -2 \theta_{13} & \left(\theta_{23}-\theta_{14}\right) \\
\left(\theta_{34}-\theta_{12}^{*}\right) & \left(\xi_{1}+\xi_{3}\right) & \left(\theta_{14}-\theta_{23}\right) & -2 \theta_{24} \\
-2 \theta_{13}^{*} & \left(\theta_{14}^{*}-\theta_{23}^{*}\right) & \left(\xi_{2}+\xi_{4}\right) & \left(\theta_{12}^{*}-\theta_{34}\right) \\
\left(\theta_{23}^{*}-\theta_{14}^{*}\right) & -2 \theta_{24}^{*} & \left(\theta_{12}-\theta_{34}^{*}\right) & \left(\xi_{1}+\xi_{3}\right)
\end{array}\right)
$$

$\left({ }^{13}\right)$ Note the order of the base states which is different from the order in $(55 c)$; here we use the order of decreasing energies of the Zeeman substates. 
To simplify the calculation, we neglect all second order terms in $\xi$ and $\theta$. Then, since the terms in $A_{\text {exch. }}^{S}, A_{\text {exch. }}^{I}$ and $E_{I S}$ are of second order in $\xi$ and $\theta$, equation (68) immediately gives the effect of a spin exchange collision within this approximation.

One easily checks that neither $\xi_{1}-\xi_{3}$ nor $\xi_{2}-\xi_{4}$ are modified during the collision (this remains true if the terms quadratic in $\xi, \theta$, are included). The reason for this is that :

$$
\begin{aligned}
& \xi_{1}-\xi_{3} \propto\left\langle F_{z}\right\rangle=\left\langle I_{z}\right\rangle+\left\langle S_{z}\right\rangle \\
& \xi_{2}-\xi_{4} \propto\left\langle S_{z}\right\rangle-\left\langle I_{z}\right\rangle
\end{aligned}
$$

and that we know that $\left\langle I_{z}\right\rangle$ and $\left\langle S_{z}\right\rangle$ (actually all components of $\langle\mathbf{I}\rangle$ and $\langle\mathbf{S}\rangle$ ) are exactly conserved by equation (49).

The time variation $(\mathrm{d} \rho / \mathrm{d} t)_{\text {spin exch. }}$ of $\rho$ due to spin exchange collisions is directly proportional to the $4 \times 4$ matrix written in the right hand side of (68) (within the linear approximation used). The coefficients of coupling between the $\xi_{\mathrm{i}}$ differ from the coefficients $\boldsymbol{A}_{i j}$ given in table 2 of reference [24]. When the main evolution of $\rho$ is due to spin exchange and when a stationary equilibrium is reached, the conditions :

$$
\left\{\begin{array}{l}
\xi_{2}=-\xi_{4} \\
\xi_{1}=-\xi_{3}
\end{array}\right.
$$

are fulfilled. They can be interpreted in two different ways : the mean values of $\left\langle I_{z}\right\rangle$ inside the two $M_{S}= \pm 1 / 2$ multiplicities are equal $\left(\xi_{1}-\xi_{2}=\xi_{4}-\xi_{3}\right)$, or the mean values of $\left\langle S_{z}\right\rangle$ inside the two $m_{I}= \pm 1 / 2$ multiplicities are equal $\left(\xi_{1}-\xi_{4}=\xi_{2}-\xi_{3}\right)$.

We also see that the various coherences are not affected in the same way by spin exchange collisions. Coherences between states having different values for both $m_{I}$ and $M_{S}$ are fully destroyed. Physically, this is because these coherences give the mean value of operators like $I_{+} S_{+}$(since $I_{+}^{2}$ and $S_{+}^{2}$ are identically zero) and because $\left\langle I_{+} S_{+}\right\rangle$is replaced by $\left\langle I_{+}\right\rangle\left\langle S_{+}\right\rangle$in a decorrelation process $\left({ }^{14}\right)$.

On the other hand, half of the coherences between states having the same value for either $m_{I}$ or $M_{S}$ is preserved. The reason is that this coherence depends on orientations like $\left\langle I_{+}\right\rangle$and $\left\langle S_{+}\right\rangle$which are completely conserved, and on alignment components like $\left\langle I_{z} S_{+}\right\rangle$for example which are destroyed (and replaced by $\left\langle I_{z}\right\rangle\left\langle S_{+}\right\rangle$). Equation (68) shows how the replacement of $\left\langle I_{x, y, z} S_{x, y, z}\right\rangle$ by $\left\langle I_{x, y, z}\right\rangle\left\langle S_{x, y, z}\right\rangle$ also produces a coupling between coherences : $\theta_{12}$ and $\theta_{34}^{*}$ are coupled with each other, as well as $\theta_{23}$ with $\theta_{14}$. With the energy levels of figure 2, these couplings are non-secular and therefore negligible in most experimental situations (nonoverlapping resonance lines). This fact is related to the inversion of the order of the $m_{I}$ levels when $M_{S}= \pm 1 / 2$ (see figure 2 : as long as the applied field is small compared to $10^{6} \mathrm{G}$, the proton precesses in the field created by $\mathrm{S}$ which changes sign for the two multiplicities 1,2 and 3,4$)$.

Let us finally assume that all coherences except $\theta_{34}$ (and $\theta_{34}^{*}$ ) are zero (this is the experimental situation in [22]), and calculate exactly all terms, including the particle identity terms. Instead of calculating $4 \times 4$ matrices $\rho$, we shall only write their $2 \times 2$ restriction $\tilde{\rho}$ to the subspace spanned by the two $|--\rangle$ and $\mid+->$ kets. We then obtain :

$$
\begin{gathered}
\left(\widetilde{\rho_{I} \otimes \rho_{S}}\right)_{\mathrm{f}}=\tilde{\rho}_{\mathrm{i}}+\left(\begin{array}{cc}
\left(\frac{1}{2}\right)\left(\xi_{2}+\xi_{4}\right)+\left(\xi_{2}+\xi_{3}\right)\left(\xi_{3}+\xi_{4}\right) & \theta_{34}\left(-\frac{1}{2}+\xi_{3}+\xi_{4}\right) \\
\theta_{34}^{*}\left(-\frac{1}{2}+\xi_{3}+\xi_{4}\right) & \left(\frac{1}{2}\right)\left(\xi_{1}+\xi_{3}\right)+\left(\xi_{1}+\xi_{4}\right)\left(\xi_{3}+\xi_{4}\right)
\end{array}\right) \\
\widetilde{\left[\rho_{S} \otimes 1_{I}, \rho_{\mathrm{i}}\right]=0} \\
\left.\widetilde{\left[\rho_{I} \otimes 1_{S}\right.}, \rho_{\mathrm{i}}\right]=\left(\begin{array}{cc}
0 & \theta_{34}\left(\xi_{2}-\xi_{1}\right) \\
-\theta_{34}^{*}\left(\xi_{2}-\xi_{1}\right) & 0
\end{array}\right)
\end{gathered}
$$

${ }^{\left({ }^{14}\right)}\left\langle I_{+}\right\rangle$and $\left\langle S_{+}\right\rangle$can be expressed as functions of $\Delta M_{S}=0$ or $\Delta m_{I}=0$ coherences respectively. The initial coherence

$$
\Delta\left(m_{I}+M_{S}\right)=2
$$

is therefore replaced by a second order product of $\Delta\left(m_{I}+M_{S}\right)=1$ coherences. 


$$
\begin{aligned}
\tilde{\rho}_{\mathrm{i}}^{2} & =\left(\begin{array}{cc}
\left(\frac{1}{4}+\xi_{3}\right)^{2}+\left|\theta_{34}\right|^{2} & \theta_{34}\left(\frac{1}{2}+\xi_{3}+\xi_{4}\right) \\
\theta_{34}^{*}\left(\frac{1}{2}+\xi_{3}+\xi_{4}\right) & \left(\frac{1}{4}+\xi_{4}\right)^{2}+\left|\theta_{34}\right|^{2}
\end{array}\right) \\
& =\operatorname{Tr}_{2}\left\{P_{\mathrm{n}} \rho_{\mathrm{i}}(1) \otimes \rho_{\mathrm{i}}(2) P_{\mathrm{e}}\right\} .
\end{aligned}
$$

In this case, the only particle identity term which is not zero is the term in $A_{\text {exch. }}^{I}$ (apparent nuclear magnetic field). The absence of any electron identity term arises from the fact that, in subspace spanned by states 3 and 4 , $S_{x}$ and $S_{y}$ are identically zero, and $S_{z}$ is proportional to the identity operator. The frequency shift of the coherence $\theta_{34}$ has two different origins : a longitudinal apparent nuclear field proportional to $\left(\xi_{1}+\xi_{4}\right)-\left(\xi_{2}+\xi_{3}\right)$ which shifts levels 3 and 4 (and therefore the eigenfrequency of $\theta_{34}$ ), and a transverse apparent nuclear field proportional to $\theta_{34}$ itself which creates a. coherence from the population difference $\xi_{4}-\xi_{3}$. The $\xi_{3}$ and $\xi_{4}$ terms of both effects actually cancel out and only a dependence in the population difference $\xi_{1}-\xi_{2}$ of the two upper levels is found at the end.

4. Conclusion. - We have seen in this article how spin exchange processes are affected by the particle identity effects. We have found various terms in the density operator evolution. The transfer terms (term in $A_{t}$ ) are probably the most important ones in practice, since they can be used experimentally to couple the polarization of different atomic species. The existence of these terms is not related to any particle identity effect. Particle indistinguishability only explains the existence of the additional terms, which correspond to more subtle effects (apparent magnetic fields, etc...). There is actually a large similarity between the terms arising from electron or nucleus identity, but in the latter case, interference effects in two opposite final collision directions must always be invoked. At very low collision energies, both kinds of identity effects should become important. As a consequence, experiments with hydrogen masers at low temperatures [25] seem well adapted to the measurements of such effects.

Acknowledgments. - It is a pleasure to thank Professors S. Crampton and D. Kleppner for useful discussions and suggestions. We also thank C. Cohen-Tannoudji and J. P. Connerade who kindly read this manuscript and let us benefit from their comments.

\section{APPENDIX}

Phase shift calculation of the coefficients $A_{t}, A_{\text {exch }}^{S}, A_{\text {exch }}^{I}$ and $E_{I S}$ - In this appendix, we recall how the various coefficients which appear in equation (45) can be evaluated from the phase shift coefficients $\delta_{l}^{\mathrm{g}}$ and $\delta_{l}^{\mathrm{u}}$ $(l=0,1,2, \ldots)$. These coefficients are associated with the two interaction potentials $V_{\mathrm{g}}(R)$ and $V_{\mathrm{u}}(R)$ between two hydrogen atoms in the ground state, fixed at a distance $R$ from each other. Similar calculations can be found in references [26] and [9] (see also references quoted in [2] and [9]).

We shall consider here four (fictitious) distinguishable particles without spin. Two of these particles, $\mathbf{e}_{1}$ and $e_{2}$, have the physical properties of electrons except spin and indistinguishability. The two others are in the same way similar to protons. For fixed positions of $\mathrm{p}_{1}$ and $\mathrm{p}_{2}$ at a distance $R$, we shall consider two stationary states of the electronic clouds. One is invariant under inversion with respect to the molecule centre (centre of gravity of $\mathrm{p}_{1}$ and $\mathrm{p}_{2}$ ) and will be called the even (or " gerade ") state :

$$
|\mathrm{G}\rangle=\frac{1}{\sqrt{2}}\left[\left|\mathrm{e}_{1}: \varphi_{\mathrm{n}_{1}} ; \mathrm{e}_{2}: \varphi_{\mathrm{n}_{2}}\right\rangle+\left|\mathrm{e}_{1}: \varphi_{\mathrm{n}_{2}} ; \mathrm{e}_{2}: \varphi_{\mathrm{n}_{1}}\right\rangle\right]
$$

The sum of the (electronic) energy of this state and of the repulsion $q^{2} / R$ between $p_{1}$ and $p_{2}$ will be called the potential energy $V_{\mathrm{g}}(R)$. The other electronic cloud stationary state has a wave function which changes sign under the same spatial inversion and will be called odd (or « ungerade.»)

$$
|\mathrm{U}\rangle=\frac{1}{\sqrt{2}}\left[\left|\mathrm{e}_{1}: \varphi_{\mathrm{n}_{1}} ; \mathrm{e}_{2}: \varphi_{\mathrm{n}_{2}}\right\rangle-\left|\mathrm{e}_{1}: \varphi_{\mathrm{n}_{2}} ; \mathrm{e}_{2}: \varphi_{\mathrm{n}_{1}}\right\rangle\right]
$$

In the same way, $V_{\mathrm{u}}(R)$ is the sum of the energy of this state and $q^{2} / R$. It is well known that, when there is some overlap between the wave functions associated to $\left|\varphi_{\mathrm{n}_{1}}\right\rangle$ and $\left|\varphi_{\mathrm{n}_{2}}\right\rangle, V_{\mathrm{g}}(R) \neq V_{\mathrm{u}}(R)$.

Using the Born-Oppenheimer approximation, it is now possible to calculate the elements of the $S$ matrix when the electrons are either in the $|G\rangle$ or in the $|U\rangle$ state. These matrix elements are :

$$
S_{\mathrm{g}}\left(\mathbf{k}_{\mathrm{f}}, \mathbf{k}_{\mathrm{i}}\right)=\left\langle\mathrm{n}_{1}: \mathbf{k}_{\mathrm{f}} ; \mathrm{n}_{2}:-\mathbf{k}_{\mathrm{f}}\left|\left\langle\mathrm{e}_{1}, \mathrm{e}_{2}: \mathrm{G}|S| \mathrm{n}_{1}: \mathbf{k}_{\mathrm{i}} ; \mathrm{n}_{2}:-\mathbf{k}_{\mathrm{i}}\right\rangle\right| \mathrm{e}_{1}, \mathrm{e}_{2}: \mathrm{G}\right\rangle
$$


and :

$$
S_{\mathrm{u}}\left(\mathbf{k}_{\mathrm{f}}, \mathbf{k}_{\mathrm{i}}\right)=\left\langle\mathrm{n}_{1}: \mathbf{k}_{\mathrm{f}} ; \mathrm{n}_{2}:-\mathbf{k}_{\mathrm{f}}\left|\left\langle\mathrm{e}_{1}, \mathrm{e}_{2}: \mathrm{U}|S| \mathrm{n}_{1}: \mathbf{k}_{\mathrm{i}} ; \mathrm{n}_{2}:-\mathbf{k}_{\mathrm{i}}\right\rangle\right| \mathrm{e}_{1}, \mathrm{e}_{2}: \mathrm{U}\right\rangle .
$$

Since $V_{\mathbf{g}}(\mathbf{R})$ and $V_{\mathrm{u}}(\mathbf{R})$ actually depend only on $|\mathbf{R}|$, it is possible to use the phase shift method to evaluate these quantities [27]. Let us note $\delta_{l}^{\mathrm{g}}$ the phase shift of the $l$ spherical wave in potential $V_{g}, \delta_{l}^{u}$ the corresponding phase shift for $V_{\mathrm{u}}$. One then has [28] :

$$
S_{\mathrm{g}}\left(\mathbf{k}_{\mathrm{f}}, \mathbf{k}_{\mathrm{i}}\right)=\frac{1}{k_{\mathrm{i}}^{2}} \delta\left(k_{\mathrm{f}}-k_{\mathrm{i}}\right) \sum_{l=0}^{\infty} \sum_{m=-l}^{+l} \mathrm{e}^{2 i \delta_{l}^{8}} Y_{l}^{m *}\left(\hat{k}_{\mathrm{f}}\right) Y_{l}^{m}\left(\hat{k}_{\mathrm{i}}\right)
$$

and, similarly :

$$
S_{\mathrm{u}}\left(\mathbf{k}_{\mathrm{f}}, \mathbf{k}_{\mathrm{i}}\right)=\frac{1}{k_{\mathrm{i}}^{2}} \delta\left(k_{\mathrm{f}}-k_{\mathrm{i}}\right) \sum_{l=0}^{\infty} \sum_{m=-l}^{l} \mathrm{e}^{2 i \delta_{l}^{u}} Y_{l}^{m *}\left(\hat{k}_{\mathrm{f}}\right) Y_{l}^{m}\left(\hat{k}_{\mathrm{i}}\right) .
$$

In these expressions, $Y_{l}^{m}$ is the spherical harmonic of order $l, m$, and $\hat{k}_{\mathrm{i}}, \hat{k}_{\mathrm{f}}$, etc... denote the angular variables associated with vectors $\mathbf{k}_{\mathrm{i}}, \mathbf{k}_{\mathrm{f}}$, etc... respectively [ $\left.\mathrm{d}^{3} k_{\mathrm{i}}=k_{\mathrm{i}}^{2} \mathrm{~d} k_{\mathrm{i}} \mathrm{d}^{2} \hat{k}_{\mathrm{i}}\right]$.

The next step of this calculation is to express the functions $S_{\mathrm{d}}$ and $S_{\mathrm{t}}$ defined in (3) and (4) in terms of $S_{\mathrm{g}}$ and $S_{\mathrm{u}}$. Actually, one sees immediately in (A.1) and (A.2) that :

$$
\begin{aligned}
S_{\mathrm{g}}\left(\mathbf{k}_{\mathrm{f}}, \mathbf{k}_{\mathrm{i}}\right) & =\frac{1}{2}\left[S_{\mathrm{d}}\left(\mathbf{k}_{\mathrm{f}}, \mathbf{k}_{\mathrm{i}}\right)+S_{\mathrm{t}}\left(\mathbf{k}_{\mathrm{f}}, \mathbf{k}_{\mathrm{i}}\right)+S_{\mathrm{t}}\left(\mathbf{k}_{\mathrm{f}}, \mathbf{k}_{\mathrm{i}}\right)+S_{\mathrm{d}}\left(\mathbf{k}_{\mathrm{f}}, \mathbf{k}_{\mathrm{i}}\right)\right] \\
& =S_{\mathrm{d}}\left(\mathbf{k}_{\mathrm{f}}, \mathbf{k}_{\mathrm{i}}\right)+S_{\mathrm{t}}\left(\mathbf{k}_{\mathrm{f}}, \mathbf{k}_{\mathrm{i}}\right)
\end{aligned}
$$

and :

$$
\begin{aligned}
S_{\mathrm{u}}\left(\mathbf{k}_{\mathrm{f}}, \mathbf{k}_{\mathrm{i}}\right) & =\frac{1}{2}\left[S_{\mathrm{d}}\left(\mathbf{k}_{\mathrm{f}}, \mathbf{k}_{\mathrm{i}}\right)-S_{\mathrm{t}}\left(\mathbf{k}_{\mathrm{f}}, \mathbf{k}_{\mathrm{i}}\right)-S_{\mathrm{t}}\left(\mathbf{k}_{\mathrm{f}}, \mathbf{k}_{\mathrm{i}}\right)+S_{\mathrm{d}}\left(\mathbf{k}_{\mathrm{f}}, \mathbf{k}_{\mathrm{i}}\right)\right] \\
& =S_{\mathrm{d}}\left(\mathbf{k}_{\mathrm{f}}, \mathbf{k}_{\mathrm{i}}\right)-S_{\mathrm{t}}\left(\mathbf{k}_{\mathrm{f}}, \mathbf{k}_{\mathrm{i}}\right) .
\end{aligned}
$$

We can therefore write :

$$
S_{\mathrm{d}}\left(\mathbf{k}_{\mathrm{f}}, \mathbf{k}_{\mathrm{i}}\right)=\frac{1}{2}\left[S_{\mathbf{g}}\left(\mathbf{k}_{\mathrm{f}}, \mathbf{k}_{\mathrm{i}}\right)+S_{\mathrm{u}}\left(\mathbf{k}_{\mathrm{f}}, \mathbf{k}_{\mathrm{i}}\right)\right]=\frac{\delta\left(k_{\mathrm{f}}-k_{\mathrm{i}}\right)}{k_{\mathbf{i}}^{2}} \sum_{l, m} \mathrm{e}^{i\left(\delta_{l}^{g}+\delta_{l}^{u}\right)} \cos \left(\delta_{l}^{\mathrm{g}}-\delta_{l}^{u}\right) Y_{l}^{m *}\left(\hat{k}_{\mathrm{f}}\right) Y_{l}^{m}\left(\hat{k}_{\mathrm{i}}\right)
$$

and :

$$
S_{\mathrm{t}}\left(\mathbf{k}_{\mathrm{f}}, \mathbf{k}_{\mathrm{i}}\right)=\frac{1}{2}\left[S_{\mathrm{g}}\left(\mathbf{k}_{\mathrm{f}}, \mathbf{k}_{\mathrm{i}}\right)-S_{\mathbf{u}}\left(\mathbf{k}_{\mathrm{f}}, \mathbf{k}_{\mathrm{i}}\right)\right]=\frac{\delta\left(k_{\mathrm{f}}-k_{\mathrm{i}}\right)}{k_{\mathrm{i}}^{2}} \sum_{l, m} \mathrm{e}^{i\left(\delta_{l}^{8}+\delta_{l}^{u}\right)} i \sin \left(\delta_{l}^{\mathrm{g}}-\delta_{l}^{\mathrm{u}}\right) Y_{l}^{m *}\left(\hat{k}_{\mathrm{f}}\right) Y_{l}^{m}\left(\hat{k}_{\mathrm{i}}\right) .
$$

These results can be brought in (10), (20), (37), (40) and then give :

$$
\begin{aligned}
A_{\mathrm{i}}=\int \mathrm{d}^{3} k_{\mathrm{i}} u\left(\mathbf{k}_{\mathrm{i}}\right) \int \mathrm{d}^{3} k_{\mathrm{i}}^{\prime} & u^{*}\left(\mathbf{k}_{\mathrm{i}}^{\prime}\right) \int k_{\mathrm{f}}^{2} \mathrm{~d} k_{\mathrm{f}} \int \mathrm{d}^{2} \hat{k}_{\mathrm{f}} \times \\
& \times\left(k_{\mathrm{i}} k_{\mathrm{i}}^{\prime}\right)^{-2} \delta\left(k_{\mathrm{f}}-k_{\mathrm{i}}\right) \delta\left(k_{\mathrm{f}}-k_{\mathrm{i}}^{\prime}\right) \sum_{l, m} \sum_{l^{\prime}, m^{\prime}} \sin \left(\delta_{l}^{\mathrm{g}}-\delta_{l}^{\mathrm{u}}\right) \sin \left(\delta_{l^{\prime}}^{\mathrm{g}}-\delta_{l^{\prime}}^{\mathrm{u}}\right) \\
& \times Y_{l}^{m *}\left(\hat{k}_{\mathrm{f}}\right) Y_{l}^{m}\left(\hat{k}_{\mathrm{i}}\right) Y_{l^{\prime}}^{m^{\prime}}\left(\hat{k}_{\mathrm{f}}\right) Y_{l^{\prime}}^{m^{\prime} *}\left(\hat{k}_{\mathrm{i}}^{\prime}\right)= \\
& \int \mathrm{d}^{3} k_{\mathrm{i}} u\left(\mathbf{k}_{\mathrm{i}}\right) \int \mathrm{d}^{3} k_{\mathrm{i}}^{\prime} u^{*}\left(\mathbf{k}_{\mathrm{i}}^{\prime}\right)\left[k_{\mathrm{i}} k_{\mathrm{i}}^{\prime}\right]^{-1} \delta\left(k_{\mathrm{i}}-k_{\mathrm{i}}^{\prime}\right) \sum_{l, m} \sin ^{2}\left(\delta_{l}^{\mathrm{g}}-\delta_{l}^{u}\right) Y_{l}^{m *}\left(\hat{k}_{\mathrm{i}}^{\prime}\right) Y_{l}^{m}\left(\hat{k}_{\mathrm{i}}\right)
\end{aligned}
$$

and, similarly :

$$
\begin{aligned}
A_{\text {exch. }}^{S}=-i C_{S}=-\frac{1}{2} \int \mathrm{d}^{3} k_{\mathrm{i}} u\left(\mathbf{k}_{\mathrm{i}}\right) \int \mathrm{d}^{3} k_{\mathrm{i}}^{\prime} u^{*}\left(\mathbf{k}_{\mathrm{i}}^{\prime}\right)\left[k_{\mathrm{i}} k_{\mathrm{i}}^{\prime}\right]^{-1} \delta\left(k_{\mathrm{i}}-k_{\mathrm{i}}^{\prime}\right) \times & \\
& \times \sum_{l, m} \sin \left[2\left(\delta_{l}^{\mathrm{g}}-\delta_{l}^{u}\right)\right] Y_{l}^{m *}\left(\hat{k}_{\mathrm{i}}^{\prime}\right) Y_{l}^{m}\left(\hat{k}_{\mathrm{i}}\right) .
\end{aligned}
$$


At this point, we can easily check that $C_{S}$ is pure imaginary. For $C_{I}$, we obtain :

$$
\begin{aligned}
A_{\text {exch. }}^{I}=-i C_{I}=-\frac{1}{2} \int \mathrm{d}^{3} k_{\mathrm{i}} u\left(\mathbf{k}_{\mathrm{i}}\right) \int \mathrm{d}^{3} k_{\mathrm{i}}^{\prime} u^{*}\left(\mathbf{k}_{\mathrm{i}}^{\prime}\right)\left[k_{\mathrm{i}} k_{\mathrm{i}}^{\prime}\right]^{-1} \delta\left(k_{\mathrm{i}}-k_{\mathbf{i}}^{\prime}\right) \times \\
\times \sum_{l, m}(-1)^{l} \sin \left[{ }^{2}\left(\delta_{l}^{\mathrm{g}}-\delta_{l}^{\mathrm{u}}\right)\right] Y_{l}^{m *}\left(\hat{k}_{\mathrm{i}}^{\prime}\right) Y_{l}^{m}\left(\hat{k}_{\mathrm{i}}\right) .
\end{aligned}
$$

We note that, in this expression, a factor $(-1)^{l}$ appears. This fact arises from the fact that in (37) $S_{\mathrm{d}}$ is calculated at $+\mathbf{k}_{\mathrm{f}},+\mathbf{k}_{\mathrm{i}}$ but $S_{\mathrm{t}}^{*}$ at $-\mathbf{k}_{\mathrm{f}}, \mathbf{k}_{\mathrm{i}}^{\prime}$. As emphasized earlier, this is characteristic of a nuclear identity interference effect. As for $D_{I S}$ and $E_{I S}$, the same type of calculation gives :

$$
\left.\begin{array}{c}
D_{I S} \\
E_{I S}
\end{array}\right\}=\int \mathrm{d}^{3} k_{\mathrm{i}} u\left(\mathbf{k}_{\mathrm{i}}\right) \int \mathrm{d}^{3} k_{\mathrm{i}}^{\prime} u^{*}\left(\mathbf{k}_{\mathrm{i}}^{\prime}\right)\left[k_{\mathrm{i}} k_{\mathrm{i}}^{\prime}\right]^{-1} \delta\left(k_{\mathrm{i}}-k_{\mathrm{i}}^{\prime}\right) \sum_{l, m}(-1)^{l}\left\{\begin{array}{c}
\cos ^{2}\left(\delta_{l}^{\mathrm{g}}-\delta_{l}^{u}\right) \\
\sin ^{2}\left(\delta_{l}^{\mathrm{g}}-\delta_{l}^{u}\right)
\end{array}\right\} Y_{l}^{m *}\left(\hat{k}_{\mathrm{i}}^{\prime}\right) Y_{l}^{m}\left(\hat{k}_{\mathrm{i}}\right) .
$$

At this stage, further simplification can be obtained by assuming particular values for the function $u\left(\mathbf{k}_{\mathrm{i}}\right)$. For example, an initial wave packet (for the relative particle) can be built so that a quasi-infinite plane wave in direction $y$ and $z$ and a well defined energy are obtained. To do this, we introduce the top hat function $\theta_{a}(x)$ which, by definition, has the value 1 when $-\frac{a}{2} \leqslant x \leqslant+\frac{a}{2}$ and zero everywhere else. We note that :

and :

$$
\lim _{a \rightarrow 0}\left\{\frac{1}{a} \theta_{a}(x)\right\}=\delta(x)
$$

$$
\int_{-\infty}^{+\infty} \mathrm{d} x\left[\theta_{a}(x)\right]^{2}=a
$$

Let us suppose that :

$$
u(\mathbf{k})=\frac{L}{\sqrt{\varepsilon}} \theta_{a=1 / L}\left(k_{x}\right) \theta_{a=1 / L}\left(k_{y}\right) \theta_{a=\varepsilon}\left(k_{z}-k_{0}\right)
$$

In this expression, the length $L$, which is supposed to be very large, is directly proportional to the lateral spread of the wave packet in directions $\mathrm{O} x$ and $\mathrm{O} y$. The factor $L / \sqrt{\varepsilon}$ insures that the normalization condition $(2 a)$ is satisfied. $\hbar k_{0}$ is the mean value of the momentum of the relative particule, $\hbar \varepsilon$ the uncertainty on this impulsion (energy spread : $\hbar^{2} k_{0} \varepsilon / m$ ). The wave function corresponding to (A.11) is :

$$
\begin{aligned}
v(x, y, z) & =\left(\frac{1}{2 \pi}\right)^{3 / 2} \int \mathrm{d}^{3} k \mathrm{e}^{i \mathbf{k . r}} u(\mathbf{k}) \\
& =\sqrt{\frac{\varepsilon}{(2 \pi)^{3}}} \frac{1}{L} \frac{\sin (x / 2 L)}{(x / 2 L)} \frac{\sin (y / 2 L)}{(y / 2 L)} \frac{\sin (z \varepsilon / 2)}{(z \varepsilon / 2)} \mathrm{e}^{i k_{0} z} .
\end{aligned}
$$

It can be compared with another normalized wave packet, having the same $z$ dependence, but constructed so that its lateral spread in directions $\mathrm{O} x$ and $\mathrm{O} y$ is exactly $L$ with a simpler $x-y$ dependence :

$$
w(x, y, z)=\frac{1}{\sqrt{L}} \theta_{a=L}(x) \frac{1}{\sqrt{L}} \theta_{a=L}(y) \sqrt{\frac{\varepsilon}{2 \pi}} \frac{\sin (z \varepsilon / 2)}{(z \varepsilon / 2)} \mathrm{e}^{i k_{0} z} .
$$

For this wave packet, the probability (integrated over all times) that the particle will cross a unit surface perpendicular to $\mathrm{O} z$ and near the origin is $1 / L^{2}$. Since :

$$
v(0,0, z)=\frac{1}{2 \pi} w(0,0, z),
$$

the same probability with the initial wave packet is $1 / 4 \pi^{2} L^{2}$. Therefore, if we calculate $A_{\mathrm{t}}$ for the function $u(\mathbf{k})$ given in (A.11), the spin exchange collision cross section is merely :

$$
\sigma_{S F}=4 \pi^{2} \dot{L^{2}} A_{\mathrm{t}}
$$


This calculation can readily be done by letting $L$ tend to infinity, $\varepsilon$ to zero and using relations (A.10). We obtain :

$$
\begin{array}{r}
A_{\mathrm{t}}=\frac{L^{2}}{\varepsilon} \int \mathrm{d}^{3} k_{\mathrm{i}} \int \mathrm{d}^{3} k_{\mathrm{i}}^{\prime} \theta_{1 / L}\left(k_{\mathrm{i} x}\right) \theta_{1 / L}\left(k_{\mathrm{i} x}^{\prime}\right) \theta_{1 / L}\left(k_{\mathrm{i} y}\right) \theta_{1 / L}\left(k_{\mathrm{i} y}^{\prime}\right) \theta_{\varepsilon}\left(k_{\mathrm{i} z}-k_{0}\right) \theta_{\varepsilon}\left(k_{\mathrm{i} z}^{\prime}-k_{0}\right)\left[k_{\mathrm{i} z} k_{\mathrm{i} z}^{\prime}\right]^{-1} \delta\left(k_{\mathrm{i} z}-k_{\mathrm{i} z}^{\prime}\right) \times \\
\sum_{l, m} \sin ^{2}\left(\delta_{l}^{\mathrm{g}}-\delta_{l}^{u}\right) Y_{l}^{m *}\left(\hat{k}_{0}\right) Y_{l}^{m}\left(\hat{k}_{0}\right)
\end{array}
$$

All integrations over $x$ and $y$ components of $\mathbf{k}_{\mathrm{i}}$ and $\mathbf{k}_{\mathrm{i}}^{\prime}$ bring in factors $1 / L$. The integrations over the $z$ components give a factor $\varepsilon / k_{0}^{2}$. Finally, the relation :

$$
\sum_{m} Y_{l}^{m *}\left(\hat{k}_{0}\right) Y_{l}^{m}\left(\hat{k}_{0}\right)=\frac{2 l+1}{4 \pi} P_{l}(1)=\frac{2 l+1}{4 \pi}
$$

can be used, and one obtains :

$$
A_{\mathrm{t}}=\frac{1}{4 \pi L^{2} k_{0}^{2}} \sum_{l}(2 l+1) \sin ^{2}\left(\delta_{l}^{\mathrm{g}}-\delta_{l}^{\mathrm{u}}\right) .
$$

The cross section $\sigma_{S F}$ is then equal to :

$$
\sigma_{S F}=4 \pi^{2} L^{2} A_{\mathrm{t}}=\frac{\pi}{k_{0}^{2}} \sum_{l}(2 l+1) \sin ^{2}\left(\delta_{l}^{\mathrm{g}}-\delta_{l}^{\mathrm{u}}\right) .
$$

Similar calculations may be performed for the other coefficients, and give :

$$
\begin{aligned}
& A_{\text {exch. }}^{S}=-i C_{S}=\frac{1}{2} \frac{1}{4 \pi L^{2} k_{0}^{2}} \sum_{l}(2 l+1) \sin \left[2\left(\delta_{l}^{\mathrm{u}}-\delta_{l}^{\mathrm{g}}\right)\right] \\
& A_{\mathrm{exch} .}^{I}=-i C_{I}=\frac{1}{2} \frac{1}{4 \pi L^{2} k_{0}^{2}} \sum_{l}(-1)^{l}(2 l+1) \sin \left[2\left(\delta_{l}^{\mathrm{u}}-\delta_{l}^{\mathrm{g}}\right)\right] .
\end{aligned}
$$

The same type of calculation applied to $(40 a)$ would give for $D_{I S}$ the result :

$$
\frac{1}{4 \pi L^{2} k_{0}^{2}} \sum_{l}(-1)^{l}(2 l+1) \cos ^{2}\left(\delta_{l}^{\mathrm{g}}-\delta_{l}^{\mathrm{u}}\right)
$$

but this series is not convergent. This is because, in the expression (A.9) of $D_{I S}$, the sum over $l$ and $m$ does not include a convergence factor like $\sin ^{2}\left(\delta_{l}^{\mathrm{g}}-\delta_{l}^{\mathrm{u}}\right)$, which tends to zero when $l \rightarrow \infty$, but a factor $\cos ^{2}\left(\delta_{l}^{\mathrm{g}}-\delta_{l}^{\mathrm{u}}\right)$. Therefore, the functions of $\hat{k}_{\mathrm{i}}^{\prime}$ and $\hat{k}_{\mathrm{i}}$ which appear in the right hand side of (A.9) are highly singular and, even when the incoming wave packet corresponds to a very large $L$ and a small $\varepsilon$, expression (A.13) has no analogue for $D_{I S}$. It is nevertheless easy to obtain a correct expression for $D_{I S}$ and $E_{I S}$, by using (40b) and (44) which shows that $D_{I S}=-E_{I S}$. One then obtains :

$$
E_{I S}=-D_{I S}=\frac{1}{4 \pi L^{2} k_{0}^{2}} \sum_{l}(-1)^{l}(2 l+1) \sin ^{2}\left(\delta_{l}^{\mathrm{g}}-\delta_{l}^{\mathrm{u}}\right) .
$$

The cross sections $\sigma_{S F}^{\prime}, \sigma_{\text {exch. }}^{S}$ and $\sigma_{\text {exch. }}^{I}$ can be defined by :

$$
\begin{aligned}
\sigma_{S F}^{\prime} & =4 \pi^{2} L^{2} E_{I S}=\frac{\pi}{k_{0}^{2}} \sum_{l}(-1)^{l}(2 l+1) \sin ^{2}\left(\delta_{l}^{\mathrm{g}}-\delta_{l}^{\mathrm{u}}\right) \\
\sigma_{\mathrm{exch} .}^{S} & =4 \pi^{2} L^{2} A_{\text {exch. }}^{S}=\frac{\pi}{2 k_{0}^{2}} \sum_{l}(2 l+1) \sin \left[2\left(\delta_{l}^{\mathrm{u}}-\delta_{l}^{\mathrm{g}}\right)\right] \\
\sigma_{\text {exch. }}^{I} & =4 \pi^{2} L^{2} A_{\text {exch. }}^{I}=\frac{\pi}{2 k_{0}^{2}} \sum_{l}(-1)^{l}(2 l+1) \sin \left[2\left(\delta_{l}^{\mathrm{u}}-\delta_{l}^{\mathrm{g}}\right)\right] .
\end{aligned}
$$

These equalities are relations (B. 3) to (B.6) of reference [9], with the notation :

$$
\left.\begin{array}{cc}
\kappa=\kappa_{S}= & A_{\text {exch. } .}^{S} / A_{\mathrm{t}}=\sigma_{\text {exch. }}^{S} / \sigma_{S F} \\
\kappa^{\prime}= & A_{\text {exch. } /}^{I} / E_{I S}=\sigma_{\text {exch. } .}^{I} / \sigma_{S F}^{\prime}
\end{array}\right\} .
$$


At low energies, when $k_{0} \rightarrow 0$, the phase shifts tend to zero and the main contribution to $A_{\mathrm{t}}, A_{\text {exch., }}^{S}, A_{\text {exch. }}^{I}$. and $E_{I S}$ comes from the $l=0$ term. Since, when $k_{0} \rightarrow 0$ :

$$
\sin \left(\delta_{0}^{\mathrm{u}}-\delta_{0}^{\mathrm{g}}\right) \propto k_{0}
$$

we see that $\sigma_{S F}$ and $\sigma_{S F}^{\prime}$ tend to finite value at vanishing collision energies, but also that both $\sigma_{\text {exch. }}^{S}$ and $\sigma_{\text {exch. }}^{I}$ tend to infinity $\left({ }^{15}\right)$.

The origin of this divergence is an interference effect in the forward direction between the direct and transfer processes. To check this point, it is convenient to write $S_{\mathrm{d}}\left(\mathbf{k}_{\mathrm{f}}, \mathbf{k}_{\mathrm{i}}\right)$ under the form :

$$
S_{\mathrm{d}}\left(\mathbf{k}_{\mathrm{f}}, \mathbf{k}_{\mathrm{i}}\right)=\delta\left(\mathbf{k}_{\mathrm{f}}-\mathbf{k}_{\mathrm{i}}\right)+\bar{S}_{\mathrm{d}}\left(\mathbf{k}_{\mathrm{f}}, \mathbf{k}_{\mathrm{i}}\right)
$$

$\left[\bar{S}_{\mathrm{d}}\right.$ is proportional to the reaction matrix]. From (A.5), one easily obtains :

$$
\bar{S}_{\mathrm{d}}\left(\mathbf{k}_{\mathrm{f}}, \mathbf{k}_{\mathrm{i}}\right)=\frac{\delta\left(k_{\mathrm{f}}-k_{\mathrm{i}}\right)}{k_{\mathrm{i}}^{2}} \sum_{l, m}\left\{\mathrm{e}^{i\left(\delta_{l}^{\mathrm{g}}+\delta_{l}^{\mathrm{u}}\right)} \cos \left(\delta_{l}^{\mathrm{g}}-\delta_{l}^{\mathrm{u}}\right)-1\right\} Y_{l}^{m *}\left(\hat{k}_{\mathrm{f}}\right) Y_{l}^{m}\left(\hat{k}_{\mathrm{i}}\right)
$$

The coefficient $\sigma_{\text {exch. }}^{S}$ is now the sum of two contributions :

with

$$
\sigma_{\text {exch. }}^{S}=\bar{\sigma}_{\text {exch. }}^{S}+\sigma_{\text {exch. }}^{S}(\text { forward })
$$

$$
\begin{gathered}
\bar{\sigma}_{\text {exch. }}^{s}=4 \pi^{2} L^{2} \bar{A}_{\text {exch. }}^{s}=-4 i \pi^{2} L^{2} \bar{C}_{S} \\
\sigma_{\text {exch. }}^{S}\left(\text { forward) }=4 \pi^{2} L^{2} A_{\text {exch. }}^{S} \text { (forward) }=-4 i \pi^{2} L^{2} C_{S}\right. \text { (forward) }
\end{gathered}
$$

In these formulas, $\bar{C}_{S}$ is obtained by replacing in (20) $S_{\mathrm{d}}$ by $\bar{S}_{\mathrm{d}}$, and $C_{S}$ (forward) by replacing $S_{\mathrm{d}}$ by $\delta\left(\mathbf{k}_{\mathrm{f}}-\mathbf{k}_{\mathrm{i}}\right.$ ). Using (A.22), we obtain by a simple calculation $\left({ }^{16}\right)$ :

$$
\begin{gathered}
\bar{\sigma}_{\text {exch. }}^{S}=\frac{\pi}{2 k_{0}^{2}} \sum_{l}(2 l+1)\left\{\sin \left[2\left(\delta_{l}^{\mathrm{u}}-\delta_{l}^{\mathrm{g}}\right)\right]-2 \mathrm{e}^{-i\left(\delta_{l}^{\mathrm{g}}+\delta_{l}^{\mathrm{u}}\right)} \sin \left(\delta_{l}^{\mathrm{u}}-\delta_{l}^{\mathrm{g}}\right)\right\} \\
\sigma_{\text {exch. }}^{S}(\text { forward })=\frac{\pi}{k_{0}^{2}} \sum_{l}(2 l+1) \mathrm{e}^{-i\left(\delta_{l}^{\mathrm{g}}+\delta_{l}^{\mathrm{u}}\right)} \sin \left(\delta_{l}^{\mathrm{u}}-\delta_{l}^{\mathrm{g}}\right)
\end{gathered}
$$

and we see that, when $k_{0} \rightarrow 0, \bar{\sigma}_{S}$ remains finite and that only $\sigma_{\text {exch. }}^{S}$ (forward) diverges [more precisely, its real part diverges, but its imaginary part remains finite].

The preceding considerations show that, in experiments at low temperatures (De Broglie wavelength of the atoms comparable to, or larger than, the potential ranges), the dominant terms in the internal variable density operator evolution, due to spin exchange collisions, are effective hamiltonian terms in $\sigma_{\text {exch. }}^{S}$ and $\sigma_{\text {exch. }}^{I}$. Spin exchange frequency shifts should become an appreciable fraction, or even exceed, the line broadening due to spin exchange collisions [in optical pumping experiments, an additional $k_{0}$ factor comes in when rate equations are calculated, since the number of collisions is proportional to the impact velocity; the line broadenings then tend to zero at low temperatures, and the shifts to constant values]. This behaviour can be checked by using numerical calculations like the ones of reference [29] (in the notation of this article, $\sigma_{S F}$ and $\sigma_{S F}^{\prime}$ are proportional to $\sigma^{+} \pm \sigma^{-}, \sigma_{\text {exch. }}^{S}$ and $\sigma_{\text {exch. }}^{I}$ to $\lambda^{+} \pm \lambda^{-}$).

It should nevertheless be kept in mind that, at very low temperatures, there is a breakdown of one of the approximations that have been made : it is no longer possible to neglect the effect of the hyperfine hamiltonian during the collision. A more precise treatment of the collision is needed, taking into account potential curves connected at long distances to several possible hyperfine internal energies of the atoms [30]. The evolution equation of the density operator must then be substantially different from the results of the present paper. Such an equation should predict a relaxation of the internal variables so that a difference of the hyperfine populations is obtained after many relaxation times (instead of a density matrix merely proportional to the unity matrix).

$\left({ }^{15}\right)$ At first sight, one could think that the same reasoning, using (A.16) and (A.17), shows that $A_{\text {exch. }}^{S}$ and $A_{\text {exch. }}^{I}$ also diverge when $k_{0} \rightarrow 0$. Actually, we know that this is impossible since the Schwarz inequality requires that $\left|A_{\text {exch. }}^{S}\right| \leqslant 1 / 2$. It must be remembered that (A.16) and (A.17) were calculated by assuming that $k_{0} L \gg 1$, so that it is not correct to use them when $k_{0}$ goes to zero, $L$ being fixed.

${ }^{16}$ ) Although their sum is real, each of the coefficients $\bar{\sigma}_{\text {exch. }}^{s}$ and $\bar{\sigma}_{\text {exch. }}$ (forward) may be complex. Their real part introduces a commutator (hamiltonian) in the equation of evolution of the spin density operator, their imaginary part an anticommutator changing the trace of the density operator. 


\section{References}

[1] See for example the references quoted in :

Cohen-Tannoudi, C. and Kastler, A., Progress in Optics, E. Wolf, editor, volume V (1966) 1.

[2] Happer, W., Rev. Mod. Phys. 44 (1972) 169.

[3] Crampton, S. B., Robinson, H. G., Kleppner, D. and Ramsey, N. F., Phys. Rev. 141 (1966) 55.

[4] Crampton, S. B., Phys. Rev. 158 (1967) 57.

[5] Vanier, J., Phys. Rev. Lett. 18 (1967) 333.

[6] Hartmann, F., J. Physique 28 (1967) 288; Phys. Lett. 24A (1967) 767.

[7] Purcell, E. M. and Field, G. B., Astrophys. J. 124 (1956) 542.

[8] Wittke, J. P. and Dicke, R. H., Phys. Rev. 103 (1956) 620.

[9] Balling, L. C., Hanson, R. J. and Pipkin, F. M., Phys. Rev. 133A (1964) 607.

[10] Glassgold, A. E., Phys. Rev. 132 (1963) 2144.

[11] Bender, P. L., Phys. Rev. 132 (1963) 2154.

[12] GrossetÊte, F., J. Physique 25 (1964) 383.

[13] Carter, G. M. and Pritchard, D. E., Phys. Rev. 5A (1972) 1225.

[14] Bender, P. L., Thesis, Princeton University (1956).

[15] Kastler, A., Mém. Soc. R. Sci. Liège I (1971) 11; Topics in Modern Physics (1971) p. 199.

[16] Crampton, S. B. and Wang, H. T. M., Phys. Rev. A 12 (1975) 1305.

[17] Cohen-Tannoudj, C., Diu, B. and Lalö̈, F., Quantum Mechanics (Wiley Interscience (New York) and Herman (Paris) publishers) 1977, or : Mécanique Quantique (Herman) 1973.
[18] Grossetête, F., J. Physique 29 (1968) 456

[19] Valberg, P., Phys. Rev. A 3 (1971) 505.

[20] Gibbs, H., Phys. Rev. A 139 (1965) 1374.

[21] Stwalley, W. C., Phys. Rev. Lett. 37 (1976) 1628.

[22] Whitehead, L. A., Hardy, W. N. and Berlinsky, A. J., Phys. Rev. Lett. 42 (1979) 1042.

[23] Crampton, S. B., Greytak, T. J., Klepprner, D., Phillips, W. D., Sмiтh, D. A. and Weinrib, A., Phys. Rev. Lett. 42 (1979) 1039.

[24] Brown, R. L., J. Res. Nat. Bur. Stand. 76A (1972) 103.

[25] Desaintfuscien, M. and Audoin, C., Phys. Rev. A 13 (1976) 2070.

[26] Dalgarno, A., Proc. R. Soc. A 262 (1961) 132.

[27] See for example : ScHIF, L. I., Quantum Mechanics, 3rd ed. (Mc Graw Hill) 1968, chap. 5, section 19.

Messiah, A., Mécanique Quantique, tome I (Dunod) or chap. VIII, Section C of reference [17].

[28] Newton, R. G., Scattering theory of waves and particles (Mc Graw Hill) 1966, chap. 11, eqs. (II.12) and (II.14)

MerzBaChER, E., Quantum Mechanics (Wiley) 1970, exercise 19.4 .

Davydov, A. S., Quantum Mechanics, 2nd ed. (Pergamon Press) 1965, eq. (109.9).

[29] Allison, A. C., Phys. Rev. A 5 (1972) 2695.

[30] Harriman, J. E., Twerdochlib, M., MaC Milleur, B. and Hirschfelder, J. O., Proc. Nat. Acad. Sci. 57 (1967) 1558. 